\title{
Excitation spectra of many-body systems by linear response: General theory and applications to trapped condensates
}

\author{
Julian Grond, ${ }^{1}$ Alexej I. Streltsov, ${ }^{1,}{ }^{*}$ Axel U. J. Lode, ${ }^{1}$ Kaspar Sakmann, ${ }^{2}$ Lorenz S. Cederbaum, ${ }^{1}$ and Ofir E. Alon ${ }^{3}$ \\ ${ }^{1}$ Theoretische Chemie, Physikalisch-Chemisches Institut, Universität Heidelberg, Im Neuenheimer Feld 229, D-69120 Heidelberg, Germany \\ ${ }^{2}$ Department of Physics, Stanford University, Stanford, California 94305, USA \\ ${ }^{3}$ Department of Physics, University of Haifa at Oranim, Tivon 36006, Israel
}

(Received 2 March 2013; published 12 August 2013)

\begin{abstract}
We derive a general linear-response many-body theory capable of computing excitation spectra of trapped interacting bosonic systems, e.g., depleted and fragmented Bose-Einstein condensates (BECs). To obtain the linear-response equations we linearize the multiconfigurational time-dependent Hartree for bosons (MCTDHB) method, which provides a self-consistent description of many-boson systems in terms of orbitals and a state vector (configurations), and is in principle numerically exact. The derived linear-response many-body theory, which we term LR-MCTDHB, is applicable to systems with interaction potentials of general form. For the special case of a $\delta$ interaction potential we show explicitly that the response matrix has a very appealing bilinear form, composed of separate blocks of submatrices originating from contributions of the orbitals, the state vector (configurations), and off-diagonal mixing terms. We further give expressions for the response weights and density response. We introduce the notion of the type of excitations, useful in the study of the physical properties of the equations. From the numerical implementation of the LR-MCTDHB equations and solution of the underlying eigenvalue problem, we obtain excitations beyond available theories of excitation spectra, such as the Bogoliubov-de Gennes (BdG) equations. The derived theory is first applied to study BECs in a one-dimensional harmonic potential. The LR-MCTDHB method contains the BdG excitations and, also, predicts a plethora of additional many-body excitations which are out of the realm of standard linear response. In particular, our theory describes the exact energy of the higher harmonic of the first (dipole) excitation not contained in the BdG theory. We next study a BEC in a very shallow one-dimensional double-well potential. We find with LR-MCTDHB low-lying excitations which are not accounted for by BdG, even though the BEC has only little fragmentation and, hence, the BdG theory is expected to be valid. The convergence of the LR-MCTDHB theory is assessed by systematically comparing the excitation spectra computed at several different levels of theory.
\end{abstract}

DOI: 10.1103/PhysRevA.88.023606

PACS number(s): 03.75.Kk, 05.30.Jp, 03.65.-w

\section{INTRODUCTION}

Correlated and fragmented Bose-Einstein condensates (BECs) and their dynamics have attracted a lot of interest in recent years [1-10]. Excitation spectra of dilute BECs have been widely studied within Bogoliubov theory [11-14], which assumes the presence of a simple condensate and only a small amount of excitations and quantum depletion.

However, in many situations fragmented BECs appear where more than one eigenvalue of the reduced one-body density matrix [15] is macroscopic [16,17]. Quite generally, when increasing interactions, depletion can become macroscopic. Xu et al. measured [18] a strong quantum depletion, which differs from the results of calculations within the Bogoliubov approach. A vast number of examples of fragmented condensates [19] is available. A very natural case for fragmentation is provided by double-well systems [20], which can exhibit on one extreme Mott-insulator-like fully fragmented states. On the other extreme, when the condensates in the wells are fully coherent, they can be described by a Gross-Pitaevskii (GP) wave function. In between these two extremes, one has nontrivial fragmented states [21,22]. In asymmetric double wells even a richer spectrum of fragmented states has been found [23]. The two-well scenarios can naturally be extended to few-well systems [24]. In optical

*Corresponding author: alexej.streltsov@pci.uni-heidelberg.de lattices the superfluid-Mott-insulator phase transition has been demonstrated experimentally [25]. Generally, in quantum systems exhibiting translational and rotational symmetry, ground-state fragmentation is likely to occur [19]. Experiments demonstrated fragmentation in metastable situations [26]. The failure of GP theory in low dimensions has been shown in $[27,28]$. Excitation frequencies measured in optical lattices with an external harmonic potential could not be understood within Bogoliubov theory [29].

In those situations where not even the ground state is of GP type, the Bogoliubov-de Gennes (BdG) equations cannot give a valid description of the excitation spectra [30]. Recently, we presented a linear-response theory for completely fragmented condensates [31], based on the best-mean-field (BMF) approach [32,33], which assumes only a single configuration. The linear-response theory for the best mean field (LR-BMF) revealed an energetic splitting of excitations for condensates in deep symmetric and asymmetric double-well potentials, which is absent in the BdG theory.

Other approaches in the literature are the direct calculation of excitations [21,34,35] using self-consistent methods and the application of the Bogoliubov approximation to lattice models [30,36]. A multimode Bogoliubov approach has been developed in [37] and extends the range of validity of BdG in double wells. Other authors also apply the linear-response approach, e.g., for the sine-Gordon model [38] and the Gutzwiller model [39-41]. 
The LR-BMF theory, as mentioned above, is formulated and applicable to fully fragmented BECs. Based on a singleconfiguration ansatz for the ground-state wave function, LRBMF provides orbital excitations only [31]. In other words, LR-BMF is applicable to BECs in a deep double well (lattice) and provides excitations to its higher bands associated with deformation of the ultracold cloud. In this paper we make a big step forward and develop a completely general and systematic linear-response theory for general systems, which can be condensed or fragmented to any degree. The starting point is represented by the multiconfigurational time-dependent Hartree for bosons (MCTDHB) method [42,43], which allows one to solve, in principle, exactly for the dynamics of a many-body system [44]. Clearly, the present theory is far more powerful than LR-BMF [31], however, for the price of utilizing a much larger Hilbert space to describe the general ground state. We term our new theory for excitation spectra LR-MCTDHB.

Until now, a large variety of new phenomena has been found using MCTDHB. Let us briefly list them. A counterintuitive regime in dynamical condensate splitting, where the final state is not the fragmented ground state, but a low-lying excited state which is coherent was discovered in [42]. The decay of selftrapping in a double well was predicted in [45], which is in stark contrast to calculations within the two-mode GP equation and the Bose-Hubbard (BH) model. The fragmentation [46] and loss of coherence [47] of attractive BECs and of repulsive ones while tunneling through a barrier to open space [48] have also been described. In [49] protocols to optimally split a BEC were addressed and in [50] the connection between depletion on the many-body level and wave chaos on the Gross-Pitaevskii level in an expanding BEC was unraveled.

Linear response of this very powerful and numerically exact method allows us to study excitation spectra in a general way, which has not been possible until now. Apart from presenting the general theory, we start with a very basic example: a BEC in a harmonic potential. Such a system was the first one which has been experimentally realized [51-53] and its excitations measured [54-56]. We now have a tool to study excitations in this system for different interaction strengths and atom numbers in a precise and systematic way. We then proceed to a very shallow double-well potential. In this regime, depletion and correlations start to build up, but a spatial separation into a left and a right system is not yet present. We examine the effect of a very small barrier on the excitation spectrum of the interacting system.

The structure of the paper is as follows. We first introduce the relevant theoretical concepts in Sec. II, such as the linearresponse theory in Sec. II A and the multiconfigurational timedependent Hartree for bosons method in Sec. II B. Section III is devoted to the theory of linear response of MCTDHB. In Sec. III A the full derivation is presented for general interactions. In Sec. III A1 the derivation is given for the linearization with respect to the orbitals, and in Sec. III A2 for the configuration-interaction (CI) coefficients. In Sec. III A3 the special case of the commonly employed $\delta$ potential is presented, and in Sec. III A4 the resulting linear-response equations are cast into a matrix form and its properties discussed. Furthermore, Sec. III B deals with the derivation of the position-space density response, and Sec. III C with an analysis tool named "type of excitation." Illustrative examples in one dimension for the here developed LR-MCTDHB theory are contained in Sec. IV. First, in Sec. IV A, a BEC in a harmonic potential is studied and, second, in Sec. IV B a BEC in a shallow double-well potential is studied. The results and physical implications are discussed. Finally, we conclude and summarize in Sec. V.

\section{THEORETICAL CONCEPTS}

\section{A. Linear-response theory}

Excitation spectra of quantum systems are of fundamental and practical interest. In principle, probing the linear response of an exact model gives access to the exact excitation spectrum $[57,58]$. As a consequence, a dynamical probing of excitations via shaking a system at different frequencies and observing the response leads to the same excitation energies no matter if it is performed in a linear regime, meaning a small driving amplitude, or in a nonlinear regime. The only difference might be the intensity of the response, i.e., some excitations are rather dark in the linear regime. However, this depends also on the choice of a suitable observable. For example, the density response of a BEC might at certain frequencies be much stronger in the momentum than in the position space; for examples see, e.g., [31].

Linear-response theory is thus a very powerful tool since it allows one to compute the resonance frequencies in a linear driving regime, and hence the exact excitation spectra, in a static framework. Clearly, the numerical efforts needed to compute the time evolution dynamics at many different frequencies is orders of magnitude larger than that needed for solving an eigenvalue problem.

However, a precise and complete spectrum can only be obtained within an exact theory. For example, the GP equation is only capable of describing the spatial dynamics of a condensed system close to the ground state, and hence its excitation frequencies are not the same as of the full manybody system. Nevertheless, this is the standard method for calculating excitation spectra of interacting bosons at zero temperature. The linear-response equations of GP are usually called Bogoliubov-de Gennes equations [12-14,59,60]. Those equations are equivalent to the equations obtained when the many-body Hamiltonian is treated in the Bogoliubov approximation $[11,61,62]$, or, up to a small correction, in the random-phase approximation [63]. This can be understood by the fact that the hole states in Bogoliubov theory lead to a damping of the excitations, which is reflected by the fact that the density in linear-response theory is, as we will see, proportional to the sum of the response amplitudes $u+v$, where $v$ is typically negative and only finite if there is quantum depletion, which is a prerequisite for hole states.

We give a short sketch of the derivation of the $\mathrm{BdG}$ equations [60]. We denote the GP equation as

$$
i \dot{\phi}=\hat{H}_{\mathrm{GP}} \phi, \quad \hat{H}_{\mathrm{GP}}=\hat{h}+\lambda|\phi|^{2},
$$

where we have the interaction strength $\lambda=\lambda_{0}(N-1)$ and $N$ is the number of bosons in the BEC. Further, we denote a small, time-dependent, and periodic perturbation in the external applied potential as $\hat{h}(\mathbf{r}) \rightarrow \hat{h}(\mathbf{r})+\delta \hat{h}(\mathbf{r}, t)$, where

$$
\delta \hat{h}(\mathbf{r}, t)=f^{+}(\mathbf{r}) e^{-i \omega t}+f^{-}(\mathbf{r}) e^{i \omega t} .
$$


Hereby, the probe frequency $\omega$ and the driving amplitudes $f^{ \pm}$ are real. Via the ansatz

$$
\sqrt{N} \phi(\mathbf{r}, t)=e^{-i \mu t}\left[\sqrt{N} \phi^{0}(\mathbf{r})+u(\mathbf{r}) e^{-i \omega t}+v^{*}(\mathbf{r}) e^{i \omega t}\right],
$$

which is an expansion around the static solution of the GP equation $\phi^{0}(\mathbf{r})$ ( $\mu$ is the chemical potential) and assuming the response amplitudes $|u\rangle$ and $|v\rangle$ to be small, the following equation is obtained:

$$
\left(\mathcal{L}_{\mathrm{BdG}}-\omega\right)\left(\begin{array}{c}
|u\rangle \\
|v\rangle
\end{array}\right)=\left(\begin{array}{c}
-\sqrt{N} f^{+}\left|\phi^{0}\right\rangle \\
\sqrt{N} f^{-}\left|\phi^{0, *}\right\rangle
\end{array}\right) .
$$

We arrive at the linear-response matrix:

$$
\mathcal{L}_{\mathrm{BdG}}=\left(\begin{array}{cc}
\hat{H}_{\mathrm{GP}}+\lambda\left|\phi^{0}\right|^{2}-\mu & \lambda\left(\phi^{0}\right)^{2} \\
-\lambda\left(\phi^{0, *}\right)^{2} & -\left(\hat{H}_{\mathrm{GP}}+\lambda\left|\phi^{0}\right|^{2}-\mu\right)
\end{array}\right),
$$

where $\hat{H}_{\mathrm{GP}}$ is constructed with $\phi^{0}$. Here and in the following we use the abbreviation $\left(a^{b}\right)^{*} \equiv a^{b, *}$. We refer to Eq. (4) (with zero right-hand side) as Bogoliubov-de Gennes equations, determining the response frequencies $\omega_{k}$ and response amplitudes $\left(\left|u^{k}\right\rangle,\left|v^{k}\right\rangle\right)^{T}$. From it we obtain response energies and response amplitudes, which are independent of the exact shape of the external perturbation. For the orbitals, which depend on the shape of the perturbation, we insert the energies and amplitudes into Eq. (3) and obtain

$$
\begin{aligned}
\phi(\mathbf{r}, t)= & e^{-i \mu t}\left\{\phi^{0}(\mathbf{r})+\frac{1}{\sqrt{N}} \sum_{k}\left[\gamma_{k} u^{k}(\mathbf{r}) e^{-i \omega t}\right.\right. \\
& \left.\left.+\gamma_{k}^{*} v^{k, *}(\mathbf{r}) e^{i \omega t}\right] /\left(\omega-\omega_{k}\right)\right\} .
\end{aligned}
$$

The response weights, which also depend on the shape of the perturbation, are given as

$$
\gamma_{k}=\sqrt{N} \int d \mathbf{r}\left[u^{k, *}(\mathbf{r}) f^{+}(\mathbf{r}) \phi^{0}(\mathbf{r})+v^{k, *}(\mathbf{r}) f^{-}(\mathbf{r}) \phi^{0, *}(\mathbf{r})\right] .
$$

In the following, a more general linear-response theory will be derived. First we discuss the underlying many-body theory which is general and, in principle, exact.

\section{B. Multiconfigurational time-dependent Hartree for bosons (MCTDHB) method}

The basic idea of the MCTDHB method is to make an ansatz for the many-body state in terms of superpositions of symmetrized states (permanents), to account for the symmetry of the bosons. The number $M$ of orbitals with which the permanents are constructed is chosen at will, e.g., (at least) two for a double-well system, etc. Then, a time-dependent variational principle is applied, and in this fashion working equations are derived for the shape of the orbitals, which make up the permanents, as well as for the coefficients (or state vector). The equations for the orbitals are coupled nonlinear equations, with nonlinearities depending on the one- and two-body reduced density matrices. They are coupled to the equation for the state vector, which is governed by the general many-body Hamiltonian in terms of the mode operators, with matrix elements depending on the orbitals. The set of equations has to be solved simultaneously. This constitutes a self-consistent (time-adaptive) approach for the condensate dynamics [42,43]. The only approximation involved is the number of modes, which can be chosen at will. Hence, for a large enough number of modes, the method gives exact results $[44,45,64]$. In imaginary time propagation MCTDHB boils down to the static, self-consistent multiconfigurational Hartree for bosons (MCHB) [21] theory. The main computational limitation is due to the state vector, which grows exponentially with the number of modes. Since this approach avoids the explicit construction of the secular matrix, the MCTDHB method can be applied to much larger systems and/or longer propagation times. Even with a smaller amount of time-adaptive modes, however, one can capture a larger amount of excitations than with fixed orbitals. For example, shape oscillations of fragmented condensates, analogous to Bogoliubov quasiparticles in a single BEC, can easily be described within MCTDHB.

Briefly, here are the ingredients of the MCTDHB theory $[42,43]$. The orbital part of the MCTDHB equations can be written in a compact form as

$$
i \rho_{i j} \frac{\partial}{\partial t}\left|\phi_{j}\right\rangle=\sum_{j=1}^{M}\left[\hat{Z}_{i j}-\mu_{i j}(t)\right]\left|\phi_{j}\right\rangle,
$$

where

$$
\hat{Z}_{i j}=\rho_{i j} \hat{h}+\sum_{s, l=1}^{M} \rho_{i s j l} \hat{W}_{s l} .
$$

The general two-body interaction operators read

$$
\hat{W}_{s l}(\mathbf{r})=\int d \mathbf{r}^{\prime} \phi_{s}^{*}\left(\mathbf{r}^{\prime}\right) \hat{W}\left(\mathbf{r}-\mathbf{r}^{\prime}\right) \phi_{l}\left(\mathbf{r}^{\prime}\right) .
$$

The one- and two-body reduced densities $[22,65,66] \rho_{i j}$ and $\rho_{i s j l}$ are given, respectively, by

$$
\rho_{i j}=\left\langle\mathbf{C}\left|\hat{a}_{i}^{\dagger} \hat{a}_{j}\right| \mathbf{C}\right\rangle, \quad \rho_{i s j l}=\left\langle\mathbf{C}\left|\hat{a}_{i}^{\dagger} \hat{a}_{s}^{\dagger} \hat{a}_{j} \hat{a}_{l}\right| \mathbf{C}\right\rangle,
$$

where $|\mathbf{C}\rangle$ stands for the coefficients (state vector).

The Lagrange multipliers account for the orthonormality of the orbitals and are given by

$$
\mu_{i j}(t)=\sum_{l=1}^{M}\left\langle\phi_{j}\left|\hat{Z}_{i l}\right| \phi_{l}\right\rangle .
$$

The coefficients' equations read

$$
i \frac{\partial \mathbf{C}(t)}{\partial t}=\mathcal{H} \mathbf{C}(t) .
$$

The many-body Hamiltonian is given as

$$
\mathcal{H}=\sum_{k, q} h_{k q} \hat{a}_{k}^{\dagger} \hat{a}_{q}+\frac{1}{2} \sum_{k, s, q, l} \hat{a}_{k}^{\dagger} \hat{a}_{s}^{\dagger} \hat{a}_{l} \hat{a}_{q} W_{k s q l},
$$

with the matrix elements

$$
h_{k q}=\int d \mathbf{r} \phi_{k}^{*}(\mathbf{r}) \hat{h} \phi_{q}(\mathbf{r})
$$

and

$$
W_{k s q l}=\int d \mathbf{r} d \mathbf{r}^{\prime} \phi_{k}^{*}(\mathbf{r}) \phi_{s}^{*}\left(\mathbf{r}^{\prime}\right) \hat{W}\left(\mathbf{r}-\mathbf{r}^{\prime}\right) \phi_{q}(\mathbf{r}) \phi_{l}\left(\mathbf{r}^{\prime}\right) .
$$


In Eqs. (13) and (14) and hereafter we follow the notation of Refs. $[64,67]$ in which the second-quantized Hamiltonian operates directly on the state vector $|\mathbf{C}\rangle$ and readdresses its components.

\section{LINEAR-RESPONSE THEORY FOR MANY-BODY SYSTEMS: LR-MCTDHB}

\section{A. Derivation}

We derive first the linear response of MCTDHB for general two-body interactions, and specify it later on to $\delta$ interactions. The orbitals' linear response is obtained by expanding around stationary ones

$$
\phi_{i}(\mathbf{r}, t) \approx \phi_{i}^{0}(\mathbf{r})+\delta \phi_{i}(\mathbf{r}, t),
$$

and the coefficients' linear response is obtained by an expansion around a stationary configuration:

$$
\mathbf{C}(t) \approx e^{-i \mathcal{E}^{0} t}\left[\mathbf{C}^{\mathbf{0}}+\delta \mathbf{C}(t)\right] .
$$

Here, $\left\{\phi_{i}^{0}(\mathbf{r}), i=1, \ldots, M\right\}$ are the orbitals, $\mathbf{C}^{\mathbf{0}}$ is the vector of coefficients, and $\mathcal{E}^{0}$ is the energy of the chosen stationary state, typically the ground state, obtained by solving the MCHB equations [21].

\section{Orbital part}

The orbital part of the MCTDHB equations including a perturbation $\delta \hat{h}(t)$ of the external potential can be written as

$$
\sum_{j=1}^{M}\left[\hat{Z}_{i j}-i \rho_{i j} \frac{\partial}{\partial t}-\mu_{i j}(t)\right]\left|\phi_{j}\right\rangle=-\sum_{j=1}^{M} \rho_{i j} \delta \hat{h}(t)\left|\phi_{j}\right\rangle,
$$

where we explicitly keep the Lagrange multipliers

$$
\mu_{i j}(t)=\sum_{l=1}^{M}\left\langle\phi_{j}\left|\hat{Z}_{i l}+\rho_{i l} \delta \hat{h}(t)\right| \phi_{l}\right\rangle .
$$

In zeroth order we obtain the orbital part of the MCHB equations for the stationary state, which we denote as

$$
\sum_{j=1}^{M}\left[\hat{Z}_{i j}^{0}-\mu_{i j}^{0}\right]\left|\phi_{j}^{0}\right\rangle=0 .
$$

All quantities herein are obtained from the stationary MCHB solution $\left\{\phi_{i}^{0}(\mathbf{r}), i=1, \ldots, M\right\}$ and $\mathbf{C}^{\mathbf{0}}$. In first order we arrive at

$$
\begin{aligned}
& \sum_{j=1}^{M}\left(\hat{Z}_{i j}^{0}-i \rho_{i j}^{0} \frac{\partial}{\partial t}-\mu_{i j}^{0}\right)\left|\delta \phi_{j}\right\rangle+\left\{\sum_{s, j, l=1}^{M} \rho_{i s j l}^{0}\left(\int d \mathbf{r}^{\prime} \phi_{s}^{0, *}\left(\mathbf{r}^{\prime}\right) \hat{W}\left(\mathbf{r}-\mathbf{r}^{\prime}\right) \delta \phi_{l}\left(\mathbf{r}^{\prime}\right)+\int d \mathbf{r}^{\prime} \delta \phi_{s}^{*}\left(\mathbf{r}^{\prime}\right) \hat{W}\left(\mathbf{r}-\mathbf{r}^{\prime}\right) \phi_{l}^{0}\left(\mathbf{r}^{\prime}\right)\right)\right. \\
& \left.+\sum_{j=1}^{M}\left[\left\langle\mathbf{C}^{0}\left|\hat{a}_{i}^{\dagger} \hat{a}_{j}\right| \delta \mathbf{C}\right\rangle+\left\langle\delta \mathbf{C}\left|\hat{a}_{i}^{\dagger} \hat{a}_{j}\right| \mathbf{C}^{0}\right\rangle\right] \hat{h}+\sum_{s, j, l=1}^{M}\left[\left\langle\mathbf{C}^{0}\left|\hat{a}_{i}^{\dagger} \hat{a}_{s}^{\dagger} \hat{a}_{j} \hat{a}_{l}\right| \delta \mathbf{C}\right\rangle+\left\langle\delta \mathbf{C}\left|\hat{a}_{i}^{\dagger} \hat{a}_{s}^{\dagger} \hat{a}_{j} \hat{a}_{l}\right| \mathbf{C}^{0}\right\rangle\right] \hat{W}_{s l}^{0}\right\}\left|\phi_{j}^{0}\right\rangle \\
& \quad-\sum_{j=1}^{M}\left[\mu_{i j}^{0}\left|\delta \phi_{j}\right\rangle+\delta \mu_{i j}(t)\left|\phi_{j}^{0}\right\rangle\right]=-\sum_{j=1}^{M} \rho_{i j}^{0} \delta \hat{h}(t)\left|\phi_{j}^{0}\right\rangle .
\end{aligned}
$$

The perturbed Lagrange multipliers are given as

$$
\begin{aligned}
\delta \mu_{i j}(t) & =\sum_{l=1}^{M} \delta\left[\left\langle\phi_{j}\left|\left(\hat{Z}_{i l}-i \rho_{i l} \frac{\partial}{\partial t}\right)\right| \phi_{l}\right\rangle\right]+\sum_{l=1}^{M} \rho_{i l}^{0}\left|\phi_{j}^{0}\right| \delta \hat{h}(t)\left|\phi_{l}^{0}\right\rangle \\
& =\sum_{l=1}^{M}\left\langle\delta \phi_{j}\left|\hat{Z}_{i l}^{0}\right| \phi_{l}^{0}\right\rangle+\left\langle\phi_{j}^{0}\right| \sum_{l=1}^{M} \delta\left(\hat{Z}_{i l}\left|\phi_{l}\right\rangle\right)+\sum_{l=1}^{M} \rho_{i l}^{0}\left\langle\phi_{j}^{0}|\delta \hat{h}(t)| \phi_{l}^{0}\right\rangle \\
& =\sum_{l=1}^{M} \mu_{i l}^{0}\left\langle\delta \phi_{j} \mid \phi_{l}^{0}\right\rangle+\left\langle\phi_{j}^{0}\right| \sum_{l=1}^{M} \delta\left(\hat{Z}_{i l}\left|\phi_{l}\right\rangle\right)+\sum_{l=1}^{M} \rho_{i l}^{0}\left\langle\phi_{j}^{0}|\delta \hat{h}(t)| \phi_{l}^{0}\right\rangle,
\end{aligned}
$$

where we used partial integration in the last step. We arrive at projected response equations

$$
\sum_{j=1}^{M}\left[\hat{P}\left(\hat{Z}_{i j}^{0}-\mu_{i j}^{0}\right)-i \rho_{i j}^{0} \frac{\partial}{\partial t}\right]\left|\delta \phi_{j}\right\rangle+\hat{P}\left\{\sum_{s, j, l=1}^{M} \rho_{i s j l}^{0} \delta \hat{W}_{s l}+\sum_{j=1}^{M} \delta \rho_{i j} \hat{h}+\sum_{s, j, l=1}^{M} \delta \rho_{i s j l} \hat{W}_{s l}\right\}\left|\phi_{j}^{0}\right\rangle=-\hat{P} \sum_{j=1}^{M} \rho_{i j}^{0} \delta \hat{h}(t)\left|\phi_{j}^{0}\right\rangle,
$$

where $\hat{P}=1-\sum_{l=1}^{M}\left|\phi_{l}^{0}\right\rangle\left\langle\phi_{l}^{0}\right|$. The perturbed densities and local interaction potentials read

$$
\begin{aligned}
\delta \rho_{i j}\left[\delta \mathbf{C}, \delta \mathbf{C}^{*}\right] & =\left\langle\mathbf{C}^{0}\left|\hat{a}_{i}^{\dagger} \hat{a}_{j}\right| \delta \mathbf{C}\right\rangle+\left\langle\delta \mathbf{C}\left|\hat{a}_{i}^{\dagger} \hat{a}_{j}\right| \mathbf{C}^{0}\right\rangle, \\
\delta \rho_{i s j l}\left[\delta \mathbf{C}, \delta \mathbf{C}^{*}\right] & =\left[\left\langle\mathbf{C}^{0}\left|\hat{a}_{i}^{\dagger} \hat{a}_{s}^{\dagger} \hat{a}_{j} \hat{a}_{l}\right| \delta \mathbf{C}\right\rangle+\left\langle\delta \mathbf{C}\left|\hat{a}_{i}^{\dagger} \hat{a}_{s}^{\dagger} \hat{a}_{j} \hat{a}_{l}\right| \mathbf{C}^{0}\right\rangle\right], \\
\delta \hat{W}_{s l}\left[\delta \phi_{l}, \delta \phi_{s}^{*}\right] & =\int d \mathbf{r}^{\prime} \phi_{s}^{0, *}\left(\mathbf{r}^{\prime}\right) \hat{W}\left(\mathbf{r}-\mathbf{r}^{\prime}\right) \delta \phi_{l}\left(\mathbf{r}^{\prime}\right)+\int d \mathbf{r}^{\prime} \delta \phi_{s}^{*}\left(\mathbf{r}^{\prime}\right) \hat{W}\left(\mathbf{r}-\mathbf{r}^{\prime}\right) \phi_{l}^{0}\left(\mathbf{r}^{\prime}\right) .
\end{aligned}
$$


We make the following ansatz for the perturbed orbitals

$$
\delta \phi_{i}(\mathbf{r}, t)=u_{i}(\mathbf{r}) e^{-i \omega t}+v_{i}^{*}(\mathbf{r}) e^{i \omega t},
$$

and for the perturbed coefficients

$$
\delta \mathbf{C}=\mathbf{C}_{\mathbf{u}} e^{-i \omega t}+\mathbf{C}_{\mathbf{v}}^{*} e^{i \omega t},
$$

where $\omega$ is the driving frequency of the external perturbation. Inserted into Eq. (24) and equating the same powers of $e^{\mp i \omega t}$ we obtain

$$
\begin{aligned}
& \hat{P}\left\{\sum_{j=1}^{M}\left(\hat{Z}_{i j}^{0}-\mu_{i j}^{0}\right)\left|u_{j}\right\rangle+\sum_{s, j, l=1}^{M} \rho_{i s j l}^{0} \delta \hat{W}_{s l}\left[u_{l}, v_{s}\right]\right. \\
& \left.\quad+\sum_{j=1}^{M} \delta \rho_{i j}\left[\mathbf{C}_{\mathbf{u}}, \mathbf{C}_{\mathbf{v}}\right] \hat{h}+\sum_{s, j, l=1}^{M} \delta \rho_{i s j l}\left[\mathbf{C}_{\mathbf{u}}, \mathbf{C}_{\mathbf{v}}\right] \hat{W}_{s l}^{0}\right\}\left|\phi_{j}^{0}\right\rangle \\
& \quad-\sum_{j=1}^{M} \omega \rho_{i j}^{0}\left|u_{j}\right\rangle=-\hat{P} \sum_{j=1}^{M} \rho_{i j}^{0} f^{+}\left|\phi_{j}^{0}\right\rangle
\end{aligned}
$$

and

$$
\begin{aligned}
& -\hat{P}^{*}\left\{\sum_{j=1}^{M}\left(\hat{Z}_{j i}^{0}-\mu_{i j}^{0, *}\right)\left|v_{j}\right\rangle+\sum_{s, j, l=1}^{M} \rho_{l j s i}^{0} \delta \hat{W}_{l s}\left[u_{s}, v_{l}\right]\right. \\
& \left.+\sum_{j=1}^{M} \delta \rho_{j i}\left[\mathbf{C}_{\mathbf{u}}, \mathbf{C}_{\mathbf{v}}\right] \hat{h}+\sum_{s, j, l=1}^{M} \delta \rho_{l j s i}\left[\mathbf{C}_{\mathbf{u}}, \mathbf{C}_{\mathbf{v}}\right] \hat{W}_{l s}^{0}\right\}\left|\phi_{j}^{0, *}\right\rangle \\
& -\sum_{j=1}^{M} \omega \rho_{j i}^{0}\left|v_{j}\right\rangle=\hat{P}^{*} \sum_{j=1}^{M} \rho_{j i}^{0} f^{-}\left|\phi_{j}^{0, *}\right\rangle
\end{aligned}
$$

\section{Coefficients part}

In zeroth order we obtain the coefficients' part of the MCHB equations for the stationary state

$$
\mathcal{H}^{0} \mathbf{C}^{0}=\mathcal{E}^{0} \mathbf{C}^{0}
$$

In first order we get

$$
i \frac{\partial \delta \mathbf{C}}{\partial t}=\left(\mathcal{H}^{0}-\mathcal{E}^{0}\right) \delta \mathbf{C}+\delta \mathcal{H} \mathbf{C}^{0},
$$

where the source term is given by

$$
\begin{aligned}
\delta \mathcal{H}= & \sum_{k, q} \delta h_{k q}\left[\delta \phi_{q}, \delta \phi_{k}^{*}, \delta \hat{h}\right] \hat{a}_{k}^{\dagger} \hat{a}_{q} \\
& +\frac{1}{2} \sum_{k, s, q, l} \hat{a}_{k}^{\dagger} \hat{a}_{s}^{\dagger} \hat{a}_{l} \hat{a}_{q} \delta W_{k s q l}\left[\delta \phi_{q}, \delta \phi_{l}, \delta \phi_{k}^{*}, \delta \phi_{s}^{*}\right] .
\end{aligned}
$$

We introduced the perturbation of the one-body matrix elements

$$
\begin{aligned}
& \delta h_{k q}\left[\delta \phi_{q}, \delta \phi_{k}^{*}, \delta \hat{h}\right] \\
& \quad=\int d \mathbf{r} \phi_{k}^{0, *}(\mathbf{r}) \hat{h} \delta \phi_{q}(\mathbf{r})+\int d \mathbf{r} \delta \phi_{k}^{*}(\mathbf{r}) \hat{h} \phi_{q}^{0}(\mathbf{r})+\delta h_{k q}^{0}[\delta \hat{h}] .
\end{aligned}
$$

Note that $\delta h_{k q}^{0}[\delta \hat{h}]=\int d \mathbf{r} \phi_{k}^{0, *}(\mathbf{r}) \delta \hat{h} \phi_{q}^{0}(\mathbf{r})$ contains the perturbation of the potential. Furthermore, the matrix elements of the two-body elements are

$$
\begin{aligned}
& \delta W_{k s q l}\left[\delta \phi_{q}, \delta \phi_{l}, \delta \phi_{k}^{*}, \delta \phi_{s}^{*}\right] \\
& =\iint d \mathbf{r} d \mathbf{r}^{\prime} \phi_{k}^{0, *}(\mathbf{r}) \phi_{s}^{0, *}\left(\mathbf{r}^{\prime}\right) \hat{W}\left(\mathbf{r}-\mathbf{r}^{\prime}\right) \delta \phi_{q}(\mathbf{r}) \phi_{l}^{0}\left(\mathbf{r}^{\prime}\right) \\
& \quad+\iint d \mathbf{r} d \mathbf{r}^{\prime} \phi_{k}^{0, *}(\mathbf{r}) \phi_{s}^{0, *}\left(\mathbf{r}^{\prime}\right) \hat{W}\left(\mathbf{r}-\mathbf{r}^{\prime}\right) \phi_{q}^{0}(\mathbf{r}) \delta \phi_{l}\left(\mathbf{r}^{\prime}\right) \\
& \quad+\iint d \mathbf{r} d \mathbf{r}^{\prime} \delta \phi_{k}^{*}(\mathbf{r}) \phi_{s}^{0, *}\left(\mathbf{r}^{\prime}\right) \hat{W}\left(\mathbf{r}-\mathbf{r}^{\prime}\right) \phi_{q}^{0}(\mathbf{r}) \phi_{l}^{0}\left(\mathbf{r}^{\prime}\right) \\
& \quad+\iint d \mathbf{r} d \mathbf{r}^{\prime} \phi_{k}^{0, *}(\mathbf{r}) \delta \phi_{s}^{*}\left(\mathbf{r}^{\prime}\right) \hat{W}\left(\mathbf{r}-\mathbf{r}^{\prime}\right) \phi_{q}^{0}(\mathbf{r}) \phi_{l}^{0}\left(\mathbf{r}^{\prime}\right) .
\end{aligned}
$$

Inserting the ansatz in Eqs. (26) and (27) into Eq. (31) and equating like powers of $e^{\mp i \omega t}$ we obtain

$$
\begin{aligned}
\omega \mathbf{C}_{\mathbf{u}}= & \left(\mathcal{H}^{0}-\mathcal{E}^{0}\right) \mathbf{C}_{\mathbf{u}}+\left[\sum_{k, q} \delta h_{k q}\left[u_{q}, v_{k}, f^{+}\right] \hat{a}_{k}^{\dagger} \hat{a}_{q}\right. \\
& \left.+\frac{1}{2} \sum_{k, s, q, l} \hat{a}_{k}^{\dagger} \hat{a}_{s}^{\dagger} \hat{a}_{l} \hat{a}_{q} \delta W_{k s q l}\left[u_{q}, u_{l}, v_{k}, v_{s}\right]\right] \mathbf{C}^{0}
\end{aligned}
$$

and

$$
\begin{aligned}
\omega \mathbf{C}_{\mathbf{v}}= & -\left(\mathcal{H}^{0, *}-\mathcal{E}^{0}\right) \mathbf{C}_{\mathbf{v}}-\left[\sum_{k, q} \delta h_{q k}\left[u_{k}, v_{q}, f^{-}\right]\left(\hat{a}_{k}^{\dagger} \hat{a}_{q}\right)^{*}\right. \\
& \left.+\frac{1}{2} \sum_{k, s, q, l}\left(\hat{a}_{k}^{\dagger} \hat{a}_{s}^{\dagger} \hat{a}_{l} \hat{a}_{q}\right)^{*} \delta W_{l q k s}\left[u_{k}, u_{s}, v_{q}, v_{l}\right]\right] \mathbf{C}^{0, *},
\end{aligned}
$$

where the following notation for the action of a combination of creation and annihilation operators $\hat{O}$ on the vector of coefficients is used: $\hat{O}^{*} \mathbf{C}^{0, *} \equiv\left\{\hat{O} \mathbf{C}^{0}\right\}^{*}$. Summarizing, Eqs. (28), (29), (35), and (36) are the set of linear-response equations for a trapped Bose system interacting via a general two-body potential $\hat{W}\left(\mathbf{r}-\mathbf{r}^{\prime}\right)$. We note that the above formulation holds for a symmetric potential $\hat{W}\left(\mathbf{r}, \mathbf{r}^{\prime}\right)$ as well.

\section{Special case: $\delta$ potential}

We now specify the two-body interactions to have the form of the widely employed $\delta$ potential $W\left(\mathbf{r}-\mathbf{r}^{\prime}\right)=\lambda_{0} \delta\left(\mathbf{r}-\mathbf{r}^{\prime}\right)$ [13]. The two-body interaction operators then simplify and read

$$
\hat{W}_{s l}(\mathbf{r})=\lambda_{0} \phi_{s}^{*}(\mathbf{r}) \phi_{l}(\mathbf{r})
$$

From this we get for the MCTDHB orbital operator [Eq. (9)]

$$
\hat{Z}_{i j}=\rho_{i j} \hat{h}+\lambda_{0} \sum_{s, l=1}^{M} \rho_{i s j l} \phi_{s}^{*} \phi_{l},
$$

and the perturbed two-body interaction operators

$$
\delta \hat{W}_{s l}\left[\delta \phi_{l}, \delta \phi_{s}^{*}\right]=\lambda_{0}\left[\phi_{s}^{0, *}(\mathbf{r}) \delta \phi_{l}(\mathbf{r})+\delta \phi_{s}^{*}(\mathbf{r}) \phi_{l}^{0}(\mathbf{r})\right] .
$$

We explicitly write out all expressions containing the orbitals' and coefficients' responses $\mathbf{u}, \mathbf{v}, \mathbf{C}_{\mathbf{u}}, \mathbf{C}_{\mathbf{v}}$. For the orbitals 
we get

$$
\begin{aligned}
\hat{P} & \left\{\sum_{j=1}^{M}\left(\hat{Z}_{i j}^{0}-\mu_{i j}^{0}\right)\left|u_{j}\right\rangle+\sum_{s, j, l=1}^{M} \lambda_{0}\left[\rho_{i s l j}^{0} \phi_{s}^{0, *}\left|u_{j}\right\rangle+\rho_{i j l s}^{0} \phi_{s}^{0}\left|v_{j}\right\rangle\right]+\sum_{l=1}^{M}\left[\left\langle\mathbf{C}^{0}\left|\hat{a}_{i}^{\dagger} \hat{a}_{l}\right| \mathbf{C}_{\mathbf{u}}\right\rangle+\left\langle\mathbf{C}^{0, *}\left|\left(\hat{a}_{i}^{\dagger} \hat{a}_{l}\right)^{T}\right| \mathbf{C}_{\mathbf{v}}\right\rangle\right] \hat{h}\right. \\
& \left.+\lambda_{0} \sum_{s, j, l=1}^{M}\left[\left\langle\mathbf{C}^{0}\left|\hat{a}_{i}^{\dagger} \hat{a}_{s}^{\dagger} \hat{a}_{l} \hat{a}_{j}\right| \mathbf{C}_{\mathbf{u}}\right\rangle+\left\langle\mathbf{C}^{0, *}\left|\left(\hat{a}_{i}^{\dagger} \hat{a}_{s}^{\dagger} \hat{a}_{l} \hat{a}_{j}\right)^{T}\right| \mathbf{C}_{\mathbf{v}}\right\rangle\right] \phi_{s}^{0, *} \phi_{j}^{0}\right\} \phi_{l}^{0}-\sum_{j=1}^{M} \omega \rho_{i j}^{0}\left|u_{j}\right\rangle=-\hat{P} \sum_{j=1}^{M} \rho_{i j}^{0} f^{+}\left|\phi_{j}^{0}\right\rangle
\end{aligned}
$$

and

$$
\begin{aligned}
& -\hat{P}^{*}\left\{\sum_{j=1}^{M}\left(\hat{Z}_{i j}^{0, *}-\mu_{i j}^{0, *}\right)\left|v_{j}\right\rangle+\sum_{s, j, l=1}^{M} \lambda_{0}\left[\rho_{i s l j}^{0, *} \phi_{s}^{0}\left|v_{j}\right\rangle+\rho_{i j l s}^{0, *} \phi_{s}^{0, *}\left|u_{j}\right\rangle\right]+\sum_{l=1}^{M}\left[\left\langle\mathbf{C}^{0, *}\left|\left(\hat{a}_{i}^{\dagger} \hat{a}_{l}\right)^{*}\right| \mathbf{C}_{\mathbf{v}}\right\rangle+\left\langle\mathbf{C}^{0}\left|\left(\hat{a}_{i}^{\dagger} \hat{a}_{l}\right)^{\dagger}\right| \mathbf{C}_{\mathbf{u}}\right\rangle\right] \hat{h}\right. \\
& \left.+\lambda_{0} \sum_{s, j, l=1}^{M}\left[\left\langle\mathbf{C}^{0, *}\left|\left(\hat{a}_{i}^{\dagger} \hat{a}_{s}^{\dagger} \hat{a}_{l} \hat{a}_{j}\right)^{*}\right| \mathbf{C}_{\mathbf{v}}\right\rangle+\left\langle\mathbf{C}^{0}\left|\left(\hat{a}_{i}^{\dagger} \hat{a}_{s}^{\dagger} \hat{a}_{l} \hat{a}_{j}\right)^{\dagger}\right| \mathbf{C}_{\mathbf{u}}\right\rangle\right] \phi_{s}^{0} \phi_{j}^{0, *}\right\} \phi_{l}^{0, *}-\sum_{j=1}^{M} \omega \rho_{j i}^{0}\left|v_{j}\right\rangle=\hat{P}^{*} \sum_{j=1}^{M} \rho_{j i}^{0} f^{-}\left|\phi_{j}^{0, *}\right\rangle,
\end{aligned}
$$

with the notation for operation on the state vector $\left\langle\mathbf{C}^{0, *}\right| \hat{O}^{T} \equiv\left\{\hat{O}\left|\mathbf{C}^{0}\right\rangle\right\}^{T}$ and $\left\langle\mathbf{C}^{0, *}\right| \hat{O}^{*} \equiv\left\{\hat{O}^{\dagger}\left|\mathbf{C}^{0}\right\rangle\right\}^{T}$, where $T$ stands for transpose. For the coefficients we find

$$
\begin{aligned}
\omega \mathbf{C}_{\mathbf{u}}= & \left(\mathcal{H}^{0}-\mathcal{E}^{0}\right) \mathbf{C}_{\mathbf{u}}+\left[\sum_{k, q}\left(\int d \mathbf{r} \phi_{k}^{0, *} \hat{h} u_{q}+\int d \mathbf{r} v_{k} \hat{h} \phi_{q}^{0}+\delta h_{k q}^{0}\left[f^{+}\right]\right) \hat{a}_{k}^{\dagger} \hat{a}_{q}\right. \\
& \left.+\lambda_{0} \sum_{k, s, q, l} \hat{a}_{k}^{\dagger} \hat{a}_{s}^{\dagger} \hat{a}_{l} \hat{a}_{q}\left(\int d \mathbf{r} \phi_{k}^{0, *} \phi_{s}^{0, *} \phi_{l}^{0} u_{q}+\int d \mathbf{r} \phi_{s}^{0, *} \phi_{q}^{0} \phi_{l}^{0} v_{k}\right)\right] \mathbf{C}^{0}
\end{aligned}
$$

and

$$
\begin{aligned}
\omega \mathbf{C}_{\mathbf{v}}= & -\left(\mathcal{H}^{0, *}-\mathcal{E}^{0}\right) \mathbf{C}_{\mathbf{v}}-\left[\sum_{k, q}\left(\int d \mathbf{r} \phi_{q}^{0, *} \hat{h} u_{k}+\int d \mathbf{r} v_{q} \hat{h} \phi_{k}^{0}+\delta h_{q k}^{0}\left[f^{-}\right]\right)\left(\hat{a}_{k}^{\dagger} \hat{a}_{q}\right)^{*}\right. \\
& \left.+\lambda_{0} \sum_{k, s, q, l}\left(\hat{a}_{k}^{\dagger} \hat{a}_{s}^{\dagger} \hat{a}_{l} \hat{a}_{q}\right)^{*}\left(\int d \mathbf{r} \phi_{s}^{0} \phi_{q}^{0, *} \phi_{l}^{0, *} u_{k}+\int d \mathbf{r} \phi_{k}^{0} \phi_{s}^{0} \phi_{l}^{0, *} v_{q}\right)\right] \mathbf{C}^{0, *}
\end{aligned}
$$

The next step is to cast the linear-response Eqs. (40)(43) in matrix form, which is done in the following subsection.

\section{Linear-response system: Matrix form and properties}

The linear-response matrix will act on the $2\left(M+N_{\text {conf }}\right)$ dimensional vector composed of the orbitals' and coefficients' response amplitudes, $\left(\mathbf{u}, \mathbf{v}, \mathbf{C}_{\mathbf{u}}, \mathbf{C}_{\mathbf{v}}\right)^{T} . N_{\text {conf }}$ is the number of configurations. Thus the general structure of the linearresponse matrix, i.e., the sizes of its submatrices, is

$$
\mathcal{L} \sim 2\left(\begin{array}{cc}
M \times M & M \times N_{\text {conf }} \\
N_{\text {conf }} \times M & N_{\text {conf }} \times N_{\text {conf }}
\end{array}\right) .
$$

$\mathcal{L}$ is given explicitly as

$$
\left(\begin{array}{ccc}
\mathcal{P}^{o} \mathcal{L}_{o} & \multicolumn{2}{c}{\mathcal{P}^{o} \mathcal{L}_{o c}} \\
& \mathcal{H}^{0}-\mathcal{E}^{0} & 0_{c} \\
\mathcal{L}_{c o} & 0_{c} & -\left(\mathcal{H}^{0, *}-\mathcal{E}^{0}\right)
\end{array}\right)
$$

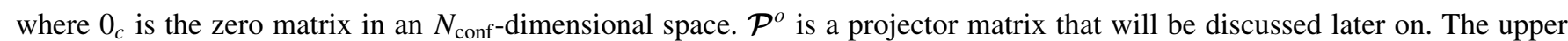
diagonal block $\mathcal{L}_{o}$ is the purely orbital part. The linear response of mean-field fragmented states, LR-BMF [31], is a special case of it, when the state vector of the many-boson system is represented by a single permanent. In the lower diagonal we have the (already linear-in terms of the coefficients) CI matrix $\mathcal{H}^{0}$ and its negative conjugate $-\mathcal{H}^{0, *}$. The hereafter obtained excitations measure the energy relative to the energy of the stationary state $\mathcal{E}^{0}$. The off diagonals account for the coupling between the orbitals' and the coefficients' response.

In the following we discuss all the involved submatrices. For the orbital part we have

$$
\mathcal{L}_{o}=\left(\begin{array}{cc}
\hat{Z}_{i j}^{0}-\mu_{i j}^{0}+\lambda_{0} \rho_{i s l j}^{0} \phi_{s}^{0, *} \phi_{l}^{0} & \lambda_{0} \rho_{i j l s}^{0} \phi_{s}^{0} \phi_{l}^{0} \\
-\lambda_{0} \rho_{i j l s}^{0, *} \phi_{s}^{0, *} \phi_{l}^{0, *} & -\left(\hat{Z}_{i j}^{0, *}-\mu_{i j}^{0, *}\right)-\lambda_{0} \rho_{i s l j}^{0, *} \phi_{s}^{0} \phi_{l}^{0, *}
\end{array}\right),
$$


where summation over doubled indices is implicitly assumed. The spaces separate the blocks for $\mathbf{u}$ and $\mathbf{v}\left(\mathbf{C}_{\mathbf{u}}\right.$ and $\mathbf{C}_{\mathbf{v}}$ below $)$, and the coupling between them. The upper right submatrix is given as

$$
\mathcal{L}_{o c}=\left(\begin{array}{cc}
\hat{h} \phi_{k}^{0}\left|\mathbf{C}_{\vec{n}_{i}^{k}}^{0}\right| \cdot+\lambda_{0} \phi_{s}^{0, *} \phi_{j}^{0} \phi_{k}^{0}\left\langle\mathbf{C}_{\vec{n}_{i s}^{k j}}^{0}\right| \cdot & \hat{h} \phi_{k}^{0}\left|\mathbf{C}_{\vec{n}_{k}^{i}}^{0, *}\right| \cdot+\lambda_{0} \phi_{s}^{0, *} \phi_{j}^{0} \phi_{k}^{0}\left\langle\mathbf{C}_{\vec{n}_{k j}^{i s}}^{0, *}\right| \cdot \\
-\hat{h} \phi_{k}^{0, *}\left|\mathbf{C}_{\vec{n}_{k}^{i}}^{0}\right| \cdot-\lambda_{0} \phi_{s}^{0} \phi_{j}^{0, *} \phi_{k}^{0, *}\left|\mathbf{C}_{\vec{n}_{k j}^{i s}}^{0}\right| \cdot & -\hat{h} \phi_{k}^{0, * *}\left|\mathbf{C}_{\vec{n}_{i}^{k}}^{0, *}\right| \cdot-\lambda_{0} \phi_{s}^{0} \phi_{j}^{0, *} \phi_{k}^{0, * *}\left|\mathbf{C}_{\vec{n}_{i s}^{k j}}^{0, *}\right| \cdot
\end{array}\right) .
$$

Each block is an $M \times N_{\text {conf }}$ matrix, i.e., all indices are summed over except for $i$, which accounts for having $M$ lines in each block. We further use in the matrix representation of the linear-response system the compact notation $\left|\mathbf{C}_{\vec{n}_{i}^{k}}^{0}\right\rangle \equiv \hat{a}_{k}^{\dagger} \hat{a}_{i}\left|\mathbf{C}^{0}\right\rangle$ and $\left|\mathbf{C}_{\bar{n}_{i s}^{k j}}^{0}\right\rangle \equiv \hat{a}_{j}^{\dagger} \hat{a}_{k}^{\dagger} \hat{a}_{s} \hat{a}_{i}\left|\mathbf{C}^{0}\right\rangle$. The lower left submatrix is given as

$$
\mathcal{L}_{c o}=\left(\begin{array}{cc}
\left|\mathbf{C}_{\vec{n}_{i}^{k}}^{0}\right\rangle \int \phi_{k}^{0, *} \hat{h} \cdot+\lambda_{0}\left|\mathbf{C}_{\vec{n}_{i l}^{k s}}^{0}\right\rangle \int \phi_{k}^{0, *} \phi_{s}^{0, *} \phi_{l}^{0 .} & \left|\mathbf{C}_{\vec{n}_{k}^{i}}^{0}\right\rangle \int \phi_{k}^{0} \hat{h} \cdot+\lambda_{0}\left|\mathbf{C}_{\vec{n}_{k l}^{i s}}^{0}\right\rangle \int \phi_{s}^{0, *} \phi_{k}^{0} \phi_{l}^{0 .} \\
-\left|\mathbf{C}_{\vec{n}_{k}^{i}}^{0 *}\right\rangle \int \phi_{k}^{0, *} \hat{h} \cdot-\lambda_{0}\left|\mathbf{C}_{\vec{n}_{k l}^{0}}^{0, *}\right\rangle \int \phi_{s}^{0} \phi_{k}^{0, *} \phi_{l}^{0, *} . & -\left|\mathbf{C}_{\vec{n}_{i}^{k}}^{0 *}\right\rangle \int \phi_{k}^{0} \hat{h} \cdot-\lambda_{0}\left|\mathbf{C}_{\vec{n}_{i l}^{k s}}^{0, *}\right\rangle \cdot \int \phi_{k}^{0} \phi_{s}^{0} \phi_{l}^{0, *} \cdot
\end{array}\right) .
$$

Here, for each $i$, an orbital (one-body function) is "lying" in each block. The dot signifies that a scalar product has to be taken with the corresponding vector element upon which the linear-response matrix acts. For $\mathcal{L}_{o c}$ it is the Euclidean scalar product in an $N_{\text {conf }}$-dimensional space. For $\mathcal{L}_{c o}$ it means integration over space.

In order to find the orthonormalization relations, we analyze the symmetry of the response matrix $\mathcal{L}$. This can be achieved by generalizing the discussion of linear-response matrices of GP [62] and BMF [31]. The orbitals' subdiagonal $\mathcal{L}_{o}$ can be analyzed in analogy to the linear-response matrix of BMF. First, one finds a time-reversal spin-flip-like symmetry

$$
\boldsymbol{\Sigma}_{\mathbf{1}}^{o} \mathcal{L}_{o} \boldsymbol{\Sigma}_{\mathbf{1}}^{o}=-\left(\mathcal{L}_{o}\right)^{*}
$$

where the matrix $\left[\Sigma_{1}^{o}\right]_{i j}=\delta_{i, j-M}+\delta_{i-M, j}(i, j=1, \ldots, 2 M)$ permutes the $i$ th and the $(M+i)$ th rows, just as the first Pauli matrix $\sigma_{1}=\left(\begin{array}{ll}0 & 1 \\ 1 & 0\end{array}\right)$ does for $M=1$. Further, we have

$$
\boldsymbol{\Sigma}_{\mathbf{3}}^{o} \mathcal{L}_{o} \boldsymbol{\Sigma}_{\mathbf{3}}^{o}=\left(\mathcal{L}_{o}\right)^{\dagger}
$$

with the matrix

$$
\left[\Sigma_{3}^{o}\right]_{i j}= \begin{cases}\delta_{i, j}, & \text { for } \quad i, j \leqslant M, \\ -\delta_{i, j}, & \text { for } \quad i, j>M .\end{cases}
$$

For the case $M=1, \Sigma_{\mathbf{3}}^{o}$ boils down to the third Pauli matrix $\sigma_{3}=\left(\begin{array}{cc}1 & 0 \\ 0 & -1\end{array}\right)$. From Eq. (49) we learn that, whenever $\left(\left|\mathbf{u}^{k}\right\rangle,\left|\mathbf{v}^{k}\right\rangle\right)^{T}$ is an eigenvector of $\mathcal{L}_{o}$ with eigenvalue $\omega_{k}$, then $\left(\left|\mathbf{v}^{k, *}\right\rangle,\left|\mathbf{u}^{k, *}\right\rangle\right)^{T}$ is an eigenvector with eigenvalue $-\left(\omega_{k}\right)^{*}$. From Eq. (50) we find that $\left(\left|\mathbf{u}^{k}\right\rangle,-\left|\mathbf{v}^{k}\right\rangle\right)^{T}$ is an eigenvector of $\left(\mathcal{L}_{o}\right)^{\dagger}$ with eigenvalue $\omega_{k}$, which allows us to construct the adjoint basis.

The same symmetries hold for the lower diagonal submatrix of $\mathcal{L}$ with respect to the matrices

$$
\boldsymbol{\Sigma}_{\mathbf{1}}^{c}=\left(\begin{array}{ll}
0_{c} & 1_{c} \\
1_{c} & 0_{c}
\end{array}\right), \quad \boldsymbol{\Sigma}_{\mathbf{3}}^{c}=\left(\begin{array}{cc}
1_{c} & 0_{c} \\
0_{c} & -1_{c}
\end{array}\right),
$$

where $1_{c}$ and $0_{c}$ are the unit and zero matrices in an $N_{\text {conf-dimensional space, respectively. For the off-diagonal }}$ submatrices of $\mathcal{L}$ we find that the diagonal blocks of $\mathcal{L}_{o c}$ are the Hermitian conjugates of the diagonals of $\mathcal{L}_{c o}$, while the upper (lower) off diagonal of $\mathcal{L}_{o c}$ is the negative Hermitian conjugate of the lower (upper) off diagonal of $\mathcal{L}_{c o}$. Hence the full linear-response matrix $\mathcal{L}$ obeys the same symmetries with respect to matrices composed of the symmetry matrices of the orbitals' and coefficients' parts

$$
\boldsymbol{\Sigma}_{\mathbf{1}}=\left(\begin{array}{cc}
\boldsymbol{\Sigma}_{\mathbf{1}}^{o} & 0_{o c} \\
0_{c o} & \boldsymbol{\Sigma}_{\mathbf{1}}^{c}
\end{array}\right), \quad \boldsymbol{\Sigma}_{\mathbf{3}}=\left(\begin{array}{cc}
\boldsymbol{\Sigma}_{\mathbf{3}}^{o} & 0_{o c} \\
0_{c o} & \boldsymbol{\Sigma}_{\mathbf{3}}^{c}
\end{array}\right) .
$$

Here, we used the zero matrices $0_{c o}$ and $0_{o c}$ with the size of the corresponding nonsquare submatrices. Finally, from those symmetries we obtain the following orthonormality condition for the response amplitudes of the orbitals and coefficients (with excitation index $k$ ):

$$
\begin{aligned}
\left\langle\mathbf{u}^{k} \mid \mathbf{u}^{k^{\prime}}\right\rangle-\left\langle\mathbf{v}^{k} \mid \mathbf{v}^{k^{\prime}}\right\rangle+\left\langle\mathbf{C}_{\mathbf{u}}{ }^{k} \mid \mathbf{C}_{\mathbf{u}}{ }^{\prime}\right\rangle-\left\langle\mathbf{C}_{\mathbf{v}}{ }^{k} \mid \mathbf{C}_{\mathbf{v}}{ }^{{ }^{\prime}}\right\rangle & =\delta_{k k^{\prime}}, \\
\left\langle\mathbf{v}^{k} \mid \mathbf{u}^{k^{\prime}, *}\right\rangle-\left\langle\mathbf{u}^{k} \mid \mathbf{v}^{k^{\prime}, *}\right\rangle+\left\langle\mathbf{C}_{\mathbf{v}} \mid \mathbf{C}_{\mathbf{u}}{ }^{k^{\prime}, *}\right\rangle-\left\langle\mathbf{C}_{\mathbf{u}}{ }^{k} \mid \mathbf{C}_{\mathbf{v}}{ }^{{ }^{\prime}, *}\right\rangle & =0 .
\end{aligned}
$$

From this we immediately see that an excitation can be either orbital-like or coefficient-like, or a mixture of both; see, for more details, Sec. III C below.

The linear-response equations read

$$
\begin{aligned}
& (\mathcal{P} \mathcal{L}-\mathcal{M} \omega)\left(\begin{array}{c}
|\mathbf{u}\rangle \\
|\mathbf{v}\rangle \\
\left|\mathbf{C}_{\mathbf{u}}\right\rangle \\
\left|\mathbf{C}_{\mathbf{v}}\right\rangle
\end{array}\right) \\
& =\mathcal{M} \mathcal{P}\left(\begin{array}{c}
-f^{+}\left|\boldsymbol{\phi}^{0}\right\rangle \\
f^{-}\left|\boldsymbol{\phi}^{0, *}\right\rangle \\
-\delta h_{k q}^{0}\left[f^{+}\right] \hat{a}_{k}^{\dagger} \hat{a}_{q}\left|\mathbf{C}^{0}\right\rangle \\
\delta h_{q k}^{0}\left[f^{-}\right]\left(\hat{a}_{k}^{\dagger} \hat{a}_{q}\right)^{*}\left|\mathbf{C}^{0, *}\right\rangle
\end{array}\right),
\end{aligned}
$$

where we again sum over double indices. The projector matrix contains twice as many projectors as the number of orbitals $M$ $(i, j=1, \ldots, 2 M)$

$$
\mathcal{P}_{i j}^{o}= \begin{cases}\hat{P}, & \text { for } i=j \leqslant M, \\ \hat{P}^{*}, & \text { for } i=j>M \\ 0 & (i \neq j)\end{cases}
$$


leading to the full matrix

$$
\mathcal{P}=\left(\begin{array}{ll}
\mathcal{P}^{o} & 0_{o c} \\
0_{c o} & 1_{c}
\end{array}\right) .
$$

Since the projector appears on both sides of Eq. (55), but not on the term proportional to the driving frequency $\omega$, the solution $\left(\mathbf{u}, \mathbf{v}, \mathbf{C}_{\mathbf{u}}, \mathbf{C}_{\mathbf{v}}\right)^{T}$ must be orthogonal to all the groundstate orbitals. We therefore can safely replace $\mathcal{P} \mathcal{L} \rightarrow \mathcal{P} \mathcal{L}$ in Eq. (55), in order to guarantee the above-discussed symmetries also in the presence of the projector. Moreover, in the linearresponse equation we have a metric

$$
\mathcal{M}=\left(\begin{array}{cccc}
\rho^{0} & & & \\
& \rho^{0, *} & & \\
& & 1_{c} & \\
& & & 1_{c}
\end{array}\right)
$$

in which the stationary reduced one-particle density matrix $\rho^{0}=\left\{\rho_{i j}^{0}\right\}$ appears. The other elements are zero. In order to render the left-hand side of Eq. (55) an eigenvalue problem, and at the same time preserve the symmetries of the corresponding matrix, we first take the square root of the metric $\mathcal{M}$ and cast the linear-response equation into the form

$$
(\overline{\mathcal{L}}-\omega)\left(\begin{array}{c}
|\overline{\mathbf{u}}\rangle \\
|\overline{\mathbf{v}}\rangle \\
\left|\overline{\mathbf{C}_{\mathbf{u}}}\right\rangle \\
\left|\overline{\mathbf{C}_{\mathbf{v}}}\right\rangle
\end{array}\right)=\left(\begin{array}{c}
-\left(\boldsymbol{\rho}^{0}\right)^{1 / 2} \mathcal{P}^{o} f^{+}\left|\boldsymbol{\phi}^{\mathbf{0}}\right\rangle \\
\left(\boldsymbol{\rho}^{0, *}\right)^{1 / 2} \mathcal{P}^{o, *} f^{-}\left|\boldsymbol{\phi}^{\mathbf{0}, *}\right\rangle \\
-\delta h_{k q}^{0}\left[f^{+}\right] \hat{a}_{k}^{\dagger} \hat{a}_{q}\left|\mathbf{C}^{0}\right\rangle \\
\delta h_{q k}^{0}\left[f^{-}\right]\left(\hat{a}_{k}^{\dagger} \hat{a}_{q}\right)^{*}\left|\mathbf{C}^{0, *}\right\rangle
\end{array}\right),
$$

where the final form of the linear-response matrix reads

$$
\overline{\mathcal{L}}=\mathcal{M}^{-1 / 2} \mathcal{P} \mathcal{L} \mathcal{P} \mathcal{M}^{-1 / 2} .
$$

Since $\mathcal{M}$ and $\mathcal{M}^{-1 / 2}$ have the same symmetry properties as $\mathcal{L}$, this is true also for $\overline{\mathcal{L}}$. Furthermore, the final form of the response amplitudes is

$$
\left(\begin{array}{c}
|\overline{\mathbf{u}}\rangle \\
|\overline{\mathbf{v}}\rangle \\
\left|\overline{\mathbf{C}_{\mathbf{u}}}\right\rangle \\
\left|\overline{\mathbf{C}_{\mathbf{v}}}\right\rangle
\end{array}\right)=\mathcal{M}^{1 / 2}\left(\begin{array}{c}
|\mathbf{u}\rangle \\
|\mathbf{v}\rangle \\
\left|\mathbf{C}_{\mathbf{u}}\right\rangle \\
\left|\mathbf{C}_{\mathbf{v}}\right\rangle
\end{array}\right)=\left(\begin{array}{c}
\left(\rho^{0}\right)^{1 / 2}|\mathbf{u}\rangle \\
\left(\rho^{0, *}\right)^{1 / 2}|\mathbf{v}\rangle \\
\left|\mathbf{C}_{\mathbf{u}}\right\rangle \\
\left|\mathbf{C}_{\mathbf{v}}\right\rangle
\end{array}\right) .
$$

We find that the same orthonormalization relations as in Eq. (54),

$$
\begin{aligned}
\left\langle\overline{\mathbf{u}}^{k} \mid \overline{\mathbf{u}}^{k^{\prime}}\right\rangle-\left\langle\overline{\mathbf{v}}^{k} \mid \overline{\mathbf{v}}^{k^{\prime}}\right\rangle+\left\langle\overline{\mathbf{C}}_{\mathbf{u}}^{k} \mid \overline{\mathbf{C}}_{\mathbf{u}}^{k^{\prime}}\right\rangle-\left\langle{\overline{\mathbf{C}_{\mathbf{v}}}}^{k} \mid{\overline{\mathbf{C}_{\mathbf{v}}}}^{k^{\prime}}\right\rangle=\delta_{k k^{\prime}}, \\
\left\langle\overline{\mathbf{v}}^{k} \mid \overline{\mathbf{u}}^{k^{\prime}, *}\right\rangle-\left\langle\overline{\mathbf{u}}^{k} \mid \overline{\mathbf{v}}^{k^{\prime}, *}\right\rangle+\left\langle\overline{\mathbf{C}}_{\mathbf{v}}{ }^{k} \mid \overline{\mathbf{C}}_{\mathbf{u}}^{k^{\prime}, *}\right\rangle-\left\langle\overline{\mathbf{C}}_{\mathbf{u}}{ }^{k} \mid{\overline{\mathbf{C}_{\mathbf{v}}}}^{k^{\prime}, *}\right\rangle=0,
\end{aligned}
$$

are satisfied. For convenience of presentation, we from now on omit the "bar" over the linear-response quantities.

In order to find the excitation energies $\omega_{k}$ in Eq. (59) one has to solve the eigenvalue problem

$$
\mathcal{P} \mathcal{L} \mathcal{P}\left(\begin{array}{c}
\left|\mathbf{u}^{k}\right\rangle \\
\left|\mathbf{v}^{k}\right\rangle \\
\left|\mathbf{C}_{\mathbf{u}}{ }^{k}\right\rangle \\
\left|\mathbf{C}_{\mathbf{v}}{ }^{k}\right\rangle
\end{array}\right)=\omega_{k}\left(\begin{array}{c}
\left|\mathbf{u}^{k}\right\rangle \\
\left|\mathbf{v}^{k}\right\rangle \\
\left|\mathbf{C}_{\mathbf{u}}{ }^{k}\right\rangle \\
\left|\mathbf{C}_{\mathbf{v}}{ }^{k}\right\rangle
\end{array}\right)
$$

(a redundant $\mathcal{P}$ is added to both sides of $\mathcal{L}$ to remind that the response is in the complementary space of the orbitals). Now we solve Eq. (59) by expanding the response vectors as well as the perturbation in the eigenvectors of $\mathcal{P} \mathcal{L} \mathcal{P}$ orthogonal to the stationary orbitals $\phi_{i}^{0}(\mathbf{r})$. The ansatz for the response amplitudes then reads

$$
\left(\begin{array}{c}
|\mathbf{u}\rangle \\
|\mathbf{v}\rangle \\
\left|\mathbf{C}_{\mathbf{u}}\right\rangle \\
\left|\mathbf{C}_{\mathbf{v}}\right\rangle
\end{array}\right)=\sum_{k} c_{k}\left(\begin{array}{c}
\left|\mathbf{u}^{k}\right\rangle \\
\left|\mathbf{v}^{k}\right\rangle \\
\left|\mathbf{C}_{\mathbf{u}}{ }^{k}\right\rangle \\
\left|\mathbf{C}_{\mathbf{v}}{ }^{k}\right\rangle
\end{array}\right)
$$

and for the perturbation

$$
\left(\begin{array}{c}
-\left(\boldsymbol{\rho}^{0}\right)^{1 / 2} \mathcal{P}^{o} f^{+}\left|\boldsymbol{\phi}^{\mathbf{0}}\right\rangle \\
\left(\boldsymbol{\rho}^{0, *}\right)^{1 / 2} \mathcal{P}^{o, *} f^{-}\left|\boldsymbol{\phi}^{\mathbf{0}, *}\right\rangle \\
-\delta h_{k q}^{0}\left[f^{+}\right] \hat{a}_{k}^{\dagger} \hat{a}_{q}\left|\mathbf{C}^{0}\right\rangle \\
\delta h_{q k}^{0}\left[f^{-}\right]\left(\hat{a}_{k}^{\dagger} \hat{a}_{q}\right)^{*}\left|\mathbf{C}^{0, *}\right\rangle
\end{array}\right)=-\sum_{k} \gamma_{k}\left(\begin{array}{c}
\left|\mathbf{u}^{k}\right\rangle \\
\left|\mathbf{v}^{k}\right\rangle \\
\left|\mathbf{C}_{\mathbf{u}}{ }^{k}\right\rangle \\
\left|\mathbf{C}_{\mathbf{v}}{ }^{k}\right\rangle
\end{array}\right) .
$$

Now $c_{k}$ and $\gamma_{k}$ have to be determined. Substituting Eqs. (64) and (65) into Eq. (59), we obtain

$$
\sum_{k} c_{k}\left(\omega_{k}-\omega\right)\left(\begin{array}{c}
\left|\mathbf{u}^{k}\right\rangle \\
\left|\mathbf{v}^{k}\right\rangle \\
\left|\mathbf{C}_{\mathbf{u}}{ }^{k}\right\rangle \\
\left|\mathbf{C}_{\mathbf{v}}{ }^{k}\right\rangle
\end{array}\right)=-\sum_{k} \gamma_{k}\left(\begin{array}{c}
\left|\mathbf{u}^{k}\right\rangle \\
\left|\mathbf{v}^{k}\right\rangle \\
\left|\mathbf{C}_{\mathbf{u}}{ }^{k}\right\rangle \\
\left|\mathbf{C}_{\mathbf{v}}{ }^{k}\right\rangle
\end{array}\right),
$$

where $\omega_{k}$ is defined in Eq. (63). From comparing the coefficients in Eq. (66) we get an expression for the $c_{k}$. Inserted into Eq. (64) leads to a solution for the response amplitudes of the form

$$
\left(\begin{array}{c}
|\mathbf{u}\rangle \\
|\mathbf{v}\rangle \\
\left|\mathbf{C}_{\mathbf{u}}\right\rangle \\
\left|\mathbf{C}_{\mathbf{v}}\right\rangle
\end{array}\right)=\sum_{k} \frac{\gamma_{k}}{\omega-\omega_{k}}\left(\begin{array}{c}
\left|\mathbf{u}^{k}\right\rangle \\
\left|\mathbf{v}^{k}\right\rangle \\
\left|\mathbf{C}_{\mathbf{u}}{ }^{k}\right\rangle \\
\left|\mathbf{C}_{\mathbf{v}}{ }^{k}\right\rangle
\end{array}\right)
$$

Reinserting the amplitudes into the ansatz for the orbitals, Eqs. (26) and (61), we arrive at the final solution for the timedependent orbitals in linear response:

$$
\begin{aligned}
\boldsymbol{\phi}(\mathbf{r}, t)= & \boldsymbol{\phi}^{\mathbf{0}}(\mathbf{r})+\left(\boldsymbol{\rho}^{0}\right)^{-1 / 2} \sum_{k}\left[\gamma_{k} \mathbf{u}^{k} e^{-i \omega t}\right. \\
& \left.+\gamma_{k}^{*} \mathbf{v}^{k, *} e^{i \omega t}\right] /\left(\omega-\omega_{k}\right),
\end{aligned}
$$

where $\boldsymbol{\phi}(\mathbf{r}, t)=\left\{\phi_{i}(\mathbf{r}, t)\right\}$ and $\boldsymbol{\phi}^{\mathbf{0}}(\mathbf{r})=\left\{\phi_{i}^{0}(\mathbf{r})\right\}$ are column vectors collecting the respective orbitals. Thus the orbitals and with them the density show the largest response at the frequencies $\omega_{k}$. Moreover, the response for a fixed frequency $\omega_{k}$ is not necessarily equally strong for all the orbitals. This is because the components of the response amplitudes $u_{j}^{k}$ and $v_{j}^{k}$ are not normalized, but rather the whole amplitude vector [see Eq. (62)]. The coefficients read to first order

$$
\begin{aligned}
\mathbf{C}(t)= & e^{-i \mathcal{E}^{0} t}\left\{\mathbf{C}^{\mathbf{0}}+\sum_{k}\left[\gamma_{k} \mathbf{C}_{\mathbf{u}}{ }^{k} e^{-i \omega t}\right.\right. \\
& \left.\left.+\gamma_{k}^{*} \mathbf{C}_{\mathbf{v}}{ }^{k, *} e^{i \omega t}\right] /\left(\omega-\omega_{k}\right)\right\} .
\end{aligned}
$$


The response weights, which quantify the intensity of the response, are given as

$$
\begin{aligned}
\gamma_{k}= & \left\langle\mathbf{u}^{k}\left|f^{+}\left(\boldsymbol{\rho}^{0}\right)^{1 / 2}\right| \boldsymbol{\phi}^{\mathbf{0}}\right\rangle+\left\langle\mathbf{v}^{k}\left|f^{-}\left(\boldsymbol{\rho}^{0, *}\right)^{1 / 2}\right| \boldsymbol{\phi}^{\mathbf{0}, *}\right\rangle \\
& +\left(\int d \mathbf{r} \phi_{i}^{0, *} f^{+} \phi_{j}^{0}\right)\left\langle\mathbf{C}_{\mathbf{u}}{ }^{k}\left|\hat{a}_{i}^{\dagger} \hat{a}_{j}\right| \mathbf{C}^{0}\right\rangle \\
& +\left(\int d \mathbf{r} \phi_{j}^{0, *} f^{-} \phi_{i}^{0}\right)\left\langle\mathbf{C}_{\mathbf{v}}{ }^{k}\left|\left(\hat{a}_{i}^{\dagger} \hat{a}_{j}\right)^{*}\right| \mathbf{C}^{0, *}\right\rangle .
\end{aligned}
$$

Similar to the orbitals [Eq. (68)], they are dominated by the largest components of the response amplitudes.

\section{B. Density oscillations}

In order to be able to calculate the density oscillations, we need first the reduced one-body density matrix $[22,65,66]$ to first order. Its elements are given by

$$
\begin{aligned}
\rho_{i j}(t)= & \left\langle\mathbf{C}(t)\left|\hat{a}_{i}^{\dagger} \hat{a}_{j}\right| \mathbf{C}(t)\right\rangle \\
\approx & \rho_{i j}^{0}+\sum_{k}\left[\gamma_{k}\left(\left\langle\mathbf{C}^{0}\left|\hat{a}_{i}^{\dagger} \hat{a}_{j}\right| \mathbf{C}_{\mathbf{u}}{ }^{k}\right\rangle+\left\langle\mathbf{C}_{\mathbf{v}}{ }^{k, *}\left|\hat{a}_{i}^{\dagger} \hat{a}_{j}\right| \mathbf{C}^{0}\right\rangle\right) e^{-i \omega t}\right. \\
& +\gamma_{k}^{*}\left(\left\langle\mathbf{C}^{0}\left|\hat{a}_{i}^{\dagger} \hat{a}_{j}\right| \mathbf{C}_{\mathbf{v}}{ }^{k, *}\right\rangle\right. \\
& \left.\left.+\left\langle\mathbf{C}_{\mathbf{u}}{ }^{k}\left|\hat{a}_{i}^{\dagger} \hat{a}_{j}\right| \mathbf{C}^{0}\right\rangle\right) e^{i \omega t}\right] /\left(\omega-\omega_{k}\right) .
\end{aligned}
$$

Together with Eq. (68), we obtain

$$
\begin{aligned}
\rho(\mathbf{r}, t)= & \sum_{i, j=1}^{M} \rho_{i j}(t) \phi_{i}^{*}(\mathbf{r}, t) \phi_{j}(\mathbf{r}, t) \\
\approx & \rho^{0}(\mathbf{r})+2 \sum_{k} \frac{1}{\omega-\omega_{k}}\left\{\operatorname{Re}\left[\gamma_{k}\right] \operatorname{Re}\left[\Delta \rho_{o}^{k}(\mathbf{r})+\Delta \rho_{c}^{k}(\mathbf{r})\right]\right. \\
& \left.-\operatorname{Im}\left[\gamma_{k}\right] \operatorname{Im}\left[\Delta \rho_{o}^{k}(\mathbf{r})+\Delta \rho_{c}^{k}(\mathbf{r})\right]\right\} \cos (\omega t) \\
& +2 \sum_{k} \frac{1}{\omega-\omega_{k}}\left\{\operatorname{Re}\left[\gamma_{k}\right] \operatorname{Im}\left[\Delta \rho_{o}^{k}(\mathbf{r})+\Delta \rho_{c}^{k}(\mathbf{r})\right]\right. \\
& \left.+\operatorname{Im}\left[\gamma_{k}\right] \operatorname{Re}\left[\Delta \rho_{o}^{k}(\mathbf{r})+\Delta \rho_{c}^{k}(\mathbf{r})\right]\right\} \sin (\omega t) .
\end{aligned}
$$

The density shows the largest response at the linear-response resonance frequencies. For simplicity, we assume real stationary orbitals $\phi_{i}^{0}(\mathbf{r})$ and reduced one-body density matrix $\rho^{0}$. We then obtain for the oscillatory part of the real-space density the orbitals' contribution

$$
\Delta \rho_{o}^{k}(\mathbf{r})=\sum_{i, j=1}^{M}\left(\boldsymbol{\rho}^{0}\right)_{i j}^{1 / 2} \phi_{i}^{0}(\mathbf{r})\left\{u_{j}^{k}(\mathbf{r})+v_{j}^{k}(\mathbf{r})\right\},
$$

and the coefficients' contribution

$$
\Delta \rho_{c}^{k}(\mathbf{r})=\left\langle\mathbf{C}^{0}\left|\hat{\rho}^{0}(\mathbf{r})\right| \mathbf{C}_{\mathbf{u}}{ }^{k}\right\rangle+\left\langle\mathbf{C}_{\mathbf{v}}{ }^{k, *}\left|\hat{\rho}^{0}(\mathbf{r})\right| \mathbf{C}^{0}\right\rangle,
$$

where we defined $\hat{\rho}^{0}(\mathbf{r})=\sum_{i, j=1}^{M} \hat{a}_{i}^{\dagger} \hat{a}_{j} \phi_{i}^{0, *}(\mathbf{r}) \phi_{j}^{0}(\mathbf{r})$.

\section{Type of excitations}

We finally reexamine the equation for the norm of the LRMCTDHB response amplitudes, Eq. (62). The left-hand side of this equation consists of the sum of the norm of the orbitals' response amplitudes and the CI response amplitudes. Hence, in order for Eq. (62) to hold, it suffices that either the orbitals' or the CI part of it is finite (and equal to one). Besides that, there can exist interesting types of mixed excitations. Let us characterize the type of the excitation by the norm of its CI response amplitude:

$$
t_{k}=\left\langle\mathbf{C}_{\mathbf{u}}{ }^{k} \mid \mathbf{C}_{\mathbf{u}}{ }^{k}\right\rangle-\left\langle\mathbf{C}_{\mathbf{v}}{ }^{k} \mid \mathbf{C}_{\mathbf{v}}{ }^{k}\right\rangle=1-\left(\left\langle\mathbf{u}^{k} \mid \mathbf{u}^{k}\right\rangle-\left\langle\mathbf{v}^{k} \mid \mathbf{v}^{k}\right\rangle\right) .
$$

Thus $t_{k}=0$ implies a purely orbital-like excitation, whereas $t_{k}=1$ indicates a purely CI-like one. Although, in general, the type of excitation is rather dependent on the basis and the number of modes used, the quantity $t_{k}$ is useful to understand the nature of the excitations, and will be analyzed in the following sections. We note that $t_{k}$ is not an observable.

\section{ILLUSTRATIVE EXAMPLES}

We now turn to the application of the LR-MCTDHB theory. We choose for simplicity the commonly used contact interparticle interaction potential. First, we study the response of many-boson systems trapped in an harmonic potential, and then turn to a more complex system-a shallow double-well potential. We obtain the excitation spectra by first calculating the ground state of the corresponding MCTDHB(M) equations; see Sec. II B. Thereafter, we explicitly construct the linear-response matrix, Eq. (60), and diagonalize it numerically solving thereby the eigenvalue problem Eq. (63). We recall here that the MCTDHB(1) level of theory is identical to the famous Gross-Pitaevskii mean field; hence the LR-MCTDHB(1) equations are the familiar LR-GP equations, often referred to in literature as the particle conserving Bogoliubov-de Gennes equations [62].

\section{A. Bose-Einstein condensates in a harmonic trap}

We start with the basic example of a BEC in a onedimensional harmonic potential

$$
V(x)=\omega_{h o}^{2} x^{2} / 2,
$$

with frequency $\omega_{h o}=\sqrt{2}$. We study the linear response of systems made of $N=100$ bosons with interparticle interaction strength $\lambda_{0}=0.01$ and of $N=10$ bosons with $\lambda_{0}=0.1$. The ground states of these systems are well described by the GP equation, since the fragmentation, i.e., the population of higher orbitals, is negligible: $0.001 \%$ for $N=100$ and $0.01 \%$ for $N=10$, respectively. These systems are characterized by the same nonlinear parameter $\lambda_{0} N=1.0$, implying that they have the same GP and, hence, the same BdG linear-response solutions. In Sec. IV A1 we compare the linear-response spectra of these systems obtained within the frameworks of the standard LR-GP (BdG) mean-field-based theory and our many-body LR-MCTDHB(2). Thereafter, in Sec. IV A2 we address the convergence of the LR-MCTDHB $(M)$ predictions for the system of $N=10$ bosons by contrasting the spectra computed at different levels $M=1,2,4,5$ of the theory. We recall that in the harmonic potential one can separate the center-of-mass ("c.m.") and relative-motion ("rel") degrees of freedom. Below, we examine the computed excitations in this context.

\section{LR-MCTDHB and LR-GP results for $N=10$ and $N=100$}

In Fig. 1 we compare the LR-GP and LR-MCTDHB(2) excitation spectra for systems of $N=100$ (lower two panels) 

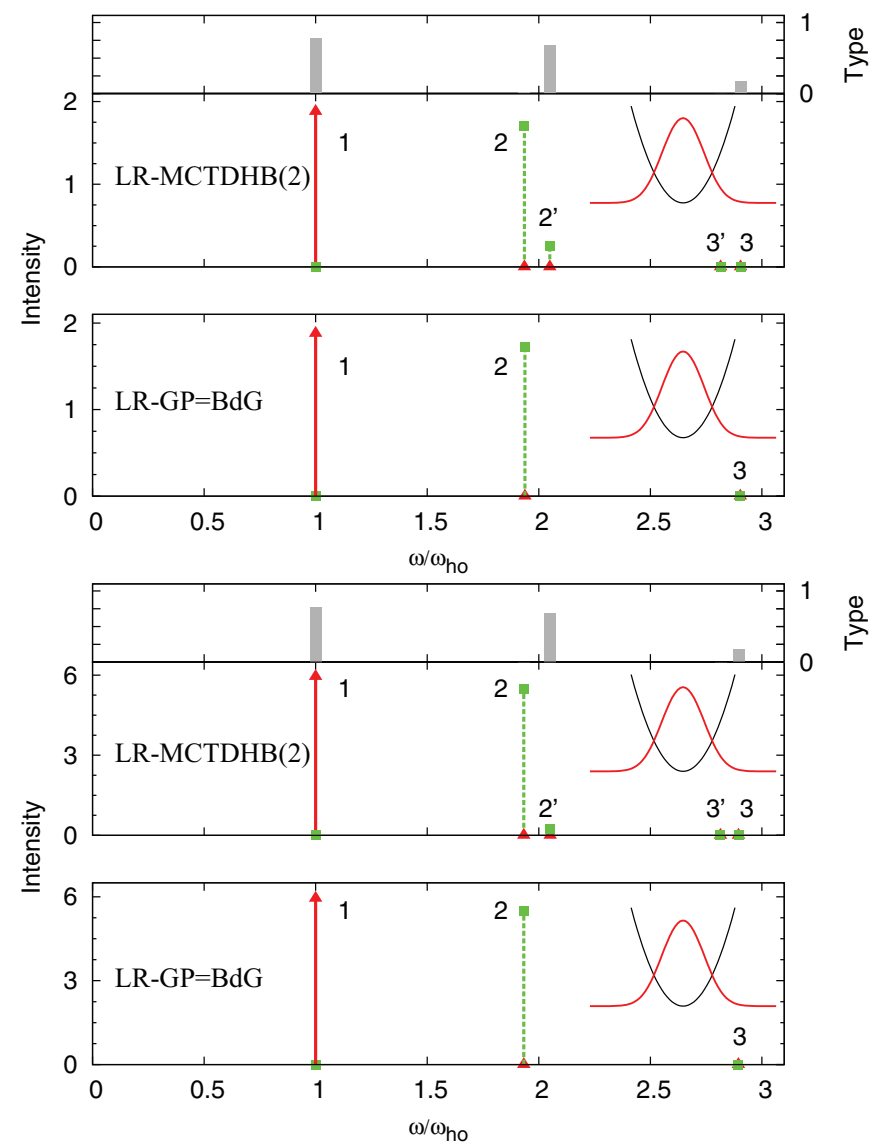

FIG. 1. (Color online) Comparison of the standard LR-GP (BdG) and LR-MCTDHB(2) linear-response spectra computed for the systems of $N=100$ (lower two panels) and $N=10$ (upper two panels) bosons trapped in a one-dimensional harmonic potential and subject to linear and quadratic perturbations. The trap potential and ground-state density (red solid line) are shown in the insets. The lower part of each panel displays excitation frequencies on the $x$ axis and response weights, Eq. (70), on the $y$ axis for linear (odd) $f^{+}=$ $f^{-}=x$ and quadratic (even) $f^{+}=f^{-}=x^{2}$ perturbations depicted by the solid (red triangle) and dashed (green squares) lines (symbols), respectively. The mean-field-based excitations, well described by both theories, are labeled 1,2,3. The many-body excitations, not describable by the BdG theory, are labeled as $2^{\prime}$ and $3^{\prime}$ (for more details, see the text and Fig. 2). The upper parts of the panels display the type of excitation $t_{k}$ computed as in Eq. (75): $t_{k}=0$ implies a purely orbital-like excitation, and $t_{k}=1$, a purely CI one. All quantities are dimensionless.

and $N=10$ (upper two panels) bosons trapped in the harmonic potential (76). The ground-state densities obtained for these systems within the GP and $\operatorname{MCTDHB}(2)$ theories are very similar and schematically shown in the insets together with the trapping potential. The $x$ axes in Fig. 1 indicate excitation energies in units of the trap frequency $\omega_{h o}$. The height of the lines or, equivalently, the position of the points along the $y$ axes, indicate response weights $\gamma_{k}$ [see Eq. (70)]. We have chosen linear $f^{+}=f^{-}=x$ and quadratic $f^{+}=f^{-}=x^{2}$ perturbations to study both odd- and even-parity excitations separately. In Fig. 1 the solid (red) lines with triangles correspond to the odd excitations, and the dashed (green) lines with squares to even ones.

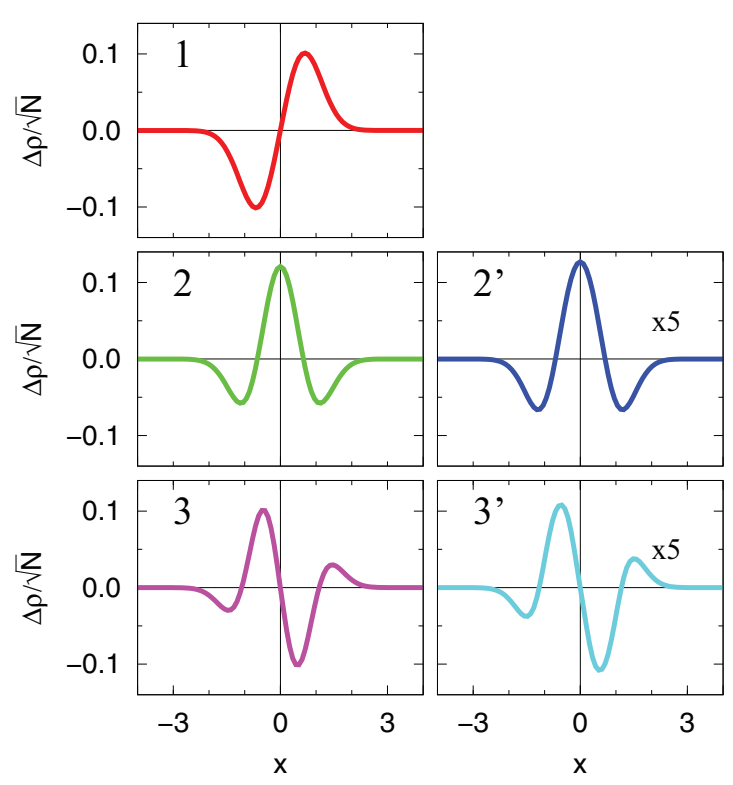

FIG. 2. (Color online) Density responses for $N=10$ bosons in a one-dimensional harmonic potential. Plotted are the real parts of the response densities $\Delta \rho(x)=\operatorname{Re}\left[\Delta \rho_{o}^{k}(x)+\Delta \rho_{c}^{k}(x)\right]$ corresponding to real-valued $\gamma_{k}$ 's; see text for details. All excitations are numerated according to the underlying nodal structures (number of nodes) in the respective response density. The LR-GP (BdG) results for the first few excitations are depicted in the left column of the figure. The LR-MCTDHB results for these excitations are quite similar and, therefore, not shown. The many-body excitations, not describable by the BdG theory, are labeled by the numbers with a prime, and magnified for better visibility. All quantities are dimensionless.

Let us first analyze the excitation spectrum of the $N=10$ system. The main observation is that the LR-MCTDHB(2) spectrum has more lines than the respective LR-GP one. Hence there are low-lying excitations not described by the BdG theory. On the other hand, the position and response weights (intensities) of the most intense lines in the spectra are well described by BdG and essentially reproduce the results of the many-body theory.

To gain more insight into the similarities and differences we plot in Fig. 2 the LR-GP and LR-MCTDHB density responses of the corresponding excitations. For their computation we have used the components of the eigenvectors, Eq. (63). The total density response is a sum of the orbital [Eq. (73)] and CI [Eq. (74)] contributions. From the derivation it is clear that the response weights $\gamma_{k}$ and response amplitudes enter Eq. (67) as a product and, therefore, there is a degree of freedom to choose the phase of the response amplitudes such that the $\gamma_{k}$ are real numbers. From now on, e.g., in Fig. 2, we plot the real parts of the response densities $\Delta \rho(x)=$ $\operatorname{Re}\left[\Delta \rho_{o}^{k}(x)+\Delta \rho_{c}^{k}(x)\right]$ corresponding to real-valued $\gamma_{k}$.

In harmonic traps the lowest-in-energy excitation corresponds to the so-called dipole oscillation and has ungerade (odd) symmetry. It has a remarkable property-it is a collective excitation of the center-of-mass motion and, due to the separability of the center-of-mass and relative motions in harmonic traps, it does not depend on the interparticle interaction. Hence its excitation energy must always be $\omega_{h o}$. We see that this excitation is nicely described by the LR-GP 
and LR-MCTDHB theories. The lowest-in-energy excitation, labeled 1 in Figs. 1 and 2, responds only to the $x$-shaped perturbation, while its contribution to the response of the $x^{2}$ perturbation is zero. In Fig. 2 one can see that the density response of the lowest-in-energy excitation has the expected one-node profile, similar to the shape of the first excited orbital of a harmonic oscillator.

The second excited state, labeled 2, has nonzero response to the $x^{2}$ perturbation only and, as seen from Fig. 2, has as expected a two-node gerade (even) profile. It originates from a single-particle excitation of the relative motion and, therefore, strongly depends on the interparticle interaction strength. This state is also well described by the LR-GP theory.

At this point the predictions of the LR-GP and LRMCTDHB theories start to deviate from each other. In addition to the widely known excitations of LR-GP, we find in our new theory excitations with finite response weights at different energies; see Fig. 1. The LR-GP predicts the third excitation at energy of about $3 \omega_{h o}$, while the LR-MCTDHB(2) theory predicts an intense excitation with energy slightly above $2 \omega_{h o}$. We label this excitation, which is not present in the LR-GP spectrum, as $2^{\prime}$ because it has zero response to $x$ perturbation, and has a two-node, gerade density-response profile very similar to the above-discussed excitation labeled 2. In Fig. 2, for better visibility, the density-response profile corresponding to excitation $2^{\prime}$ is magnified five times.

To get a deeper insight into the nature of this excited state, we analyze the type of LR-MCTDHB excitation involved. Since any eigenvector of the response matrix is normalized, by using Eq. (75) one can compute the relative contribution of the orbitals' and CI components, $t_{k}$. We recall for reference that all LR-GP excitations are orbital-like, i.e., $t_{k}=0$. In the upper panel of Fig. 1 we plot by the gray bars the results of such a decomposition analysis for each LR-MCTDHB excitation. We will discuss the general usefulness of these quantities in detail in Sec. IV A2; here we use them first to analyze the nature of the $2^{\prime}$ excitation. In Fig. 1 one can see that the $2^{\prime}$ excitation is CI dominated. Moreover, it has a two-hole-two-particle $(2 h-2 p)$ structure, i.e., two atoms are excited from the initially condensed GP-like state, making thereby two holes $(2 h)$ there, to the lowest excited orbital, thus creating two particles $(2 p)$ in this orbital. This state, therefore, represents a higher harmonic of the dipole excitation in a harmonic potential. Here we show that, with a many-body ansatz for the ground state, our LRMCTDHB theory provides direct access to this excitation. We discuss the convergence of this excitation below.

In general, all $2 h-2 p$ excitations have smaller response weights and smaller response of the position-space densities, compared to their one-hole-one-particle $(1 h-1 p)$ counterparts. To prove this statement let us consider a trapped noninteracting system, its ground $|N, 0\rangle$, and its lowest-in-energy $1 h-1 p$ and $2 h-2 p$ excited states $|N-1,1\rangle$ and $|N-2,2\rangle$. The response of the system to a perturbation $f(x)$ is defined by a superposition of individual contributions from every excited state available in the system with weight $\gamma$. For a noninteracting system the weight of the $1 h-1 p$ excitation $|N-1,1\rangle$ can be computed as $\gamma=\left\langle N, 0\left|\hat{a}_{1}^{\dagger} \hat{a}_{2}\right| N-1,1\right\rangle \int \psi_{1}^{*}(x) f(x) \psi_{0}(x) d x=$ $\sqrt{N} \int \psi_{1}^{*}(x) f(x) \psi_{0}(x) d x$. Here, $\psi_{i}(x)$ are the eigenfunctions of the trap potential. We see that the intensity of any $1 h-1 p$ excitation is proportional to the square root of the number of particles $\sqrt{N}$ times a transition integral. This result should be contrasted with that of a $2 h-2 p$ excitation which contributes with zero intensity because $\left\langle N, 0\left|\hat{a}_{1}^{\dagger} \hat{a}_{2}\right| N-2,2\right\rangle \equiv 0$. We can thus conclude that the nonzero linear-response weight of the $2^{\prime}$ excitation is solely due to interparticle interactions.

Let us discuss the last, higher-energy part of the spectra presented in Fig. 1. Our LR-MCTDHB(2) theory predicts two lines, marked as $3^{\prime}$ and 3 , respectively, while the LRGP predicts only one line labeled as 3 . This attribution is based on one and the same analysis scheme as done above, namely, that the excitations are numerated according to the underlying nodal structures (number of nodes) in the respective response density. From Fig. 2 it is clearly seen that the density response of these lines has a three-node, ungerade shape. As a consequence, these states do not respond to the $x^{2}$ perturbation. The excited state marked as 3 is presented in both the LR-GP and LR-MCTDHB computations and can be visualized as a $1 h-1 p$ excitation of one boson from the ground to a third excited orbital. The additional excitation, labeled as $3^{\prime}$, found by LR-MCTDHB is a non-mean-field-based state, because it corresponds to a two-boson excitation from the ground state. It has an interesting structure-one boson is excited to the lowest one-node ungerade orbital, and the second boson is simultaneously transferred to the lowest-in-energy two-node gerade orbital. We discuss the convergence of this excitation below.

Finally, let us compare the LR-MCTDHB(2) and LR-GP spectra for the system made of $N=100$ particles. We have chosen the interparticle interaction such that the nonlinear parameter $\lambda_{0} N=1$ is the same as in the above-discussed case of $N=10$ bosons. This choice guarantees that the GP and LR-GP solutions for both systems are essentially the same. By comparing the LR-GP spectra for $N=10$ and $N=100$ depicted in Fig. 1 one can see that, indeed, the positions of the spectral lines and their relative intensities are the same in both cases. The only difference is the absolute value of the intensities, which, as it follows from the above discussion, are proportional to the square root of the number of particles $\sqrt{N}$ times a transition integral. The LR-MCTDHB results for the mean-field-based $(1 h-1 p)$ excitations, labeled as 1,2,3 are quite similar to the LR-GP ones-their absolute intensities follow the same $\sqrt{N}$ scaling law. The main difference is found in the intensities of the $2 h-2 p$ excitations. Indeed, one can clearly see from Fig. 1 that the relative intensity of the $2^{\prime}$ line in the $N=100$ spectrum is smaller than that in the $N=10$ case. This means that, with the same one-body perturbing field and for the same interaction parameter $\lambda_{0} N$, it is more difficult to excite the $2^{\prime}$ excitation in a system with a larger number of particles.

Summarizing, we have contrasted and analyzed the predictions of the standard LR-GP (BdG) and our many-body LR$\operatorname{MCTDHB}(2)$ theories in the situation where the initial state is essentially completely condensed, i.e., the GP and LR-GP theories are believed to provide adequate descriptions. The many-body LR-MCTDHB theory contains the mean-fieldbased excitations, and also predicts additional many-body excited states which are out of the realm of the mean-field linear response. The response of these many-body excited 

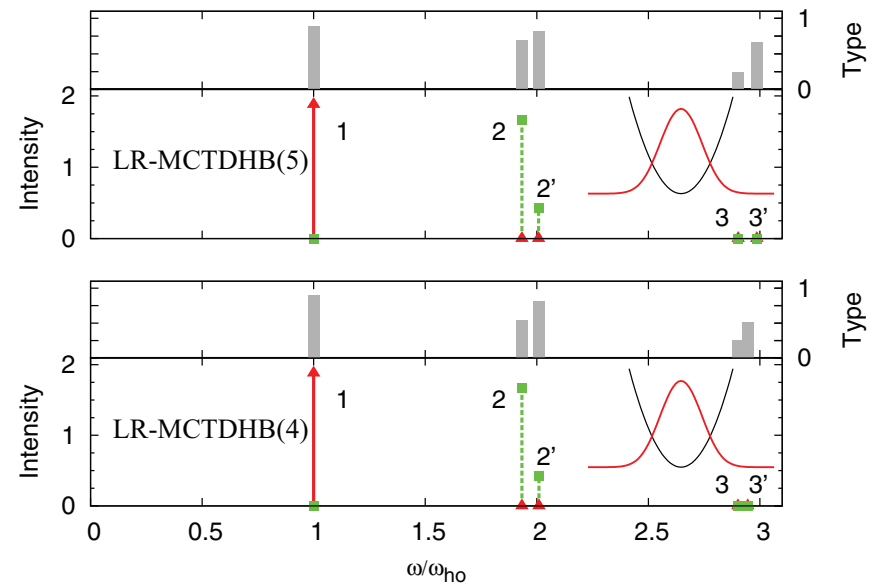

FIG. 3. (Color online) Convergence of the LR-MCTDHB $(M)$ theory. Comparison of the linear-response spectra for $N=10$ bosons in a one-dimensional harmonic potential subject to linear and quadratic perturbations computed at the LR-MCTDHB(4) and LR-MCTDHB(5) levels of theory. The depicted response weights and state characterizations are done in the same way as in Fig. 1. The main observation is that the lowest-in-energy excitations are numerically converged already at a low level of the LR-MCTDHB $(M)$ theory. By increasing $M$ we improve the quality of description and converge the higher-energy excitations as well; see text, Table I, and Fig. 4 for further details. All quantities are dimensionless.

states to the perturbations studied strongly depends on the details of the system - on the interparticle interactions and on the total number of atoms.

\section{Including more modes $(N=10$ and $M=4,5)$}

Now, having investigated many-body excitations in a harmonic potential, we would like to address the question of how reliable the obtained predictions are. To answer this question we wish to study the convergence of the results. Namely, we study now how the inclusion of more orbitals in the LR-MCTDHB theory will change the linear-response results. Since computation of the linear response with more than two orbitals is a more involved numerical task, we restrict ourselves here to studying a smaller system with $N=10$ bosons only. In Fig. 3 we present the linear-response spectra computed for this system at the LR-MCTDHB(4) and LR-MCTDHB(5) levels of theory.

It is worthwhile to mention that the $\operatorname{MCTDHB}(M)$ theory is capable of providing a numerically exact description of the statics and dynamics of interacting many-boson systems [44, $45,64]$. So, by increasing the number $M$ of the self-consistent (time-adaptive) modes used in the computations we increase the quality of description of the ground-state wave function. Since the ground-state wave function is used by the linearresponse theory to access the excited states, the quality of the excited states also improves with increasing $M$. Indeed, by comparing the LR-MCTDHB $(M), M=1,2,4,5$ excitation spectra depicted in Figs. 1 and 3, we observe the convergence of the results. Let us discuss the convergence of the excitations. To aid our discussion and to clearly visualize the convergence of excitations, we collect from Figs. 1 and 3 in Table I the frequencies of the $1,2,2^{\prime}, 3$, and $3^{\prime}$ excitations as a function of $M$. The intensities of the lines follow a similar trend concerning the convergence.

The first two excitations, 1 and 2, are available already at the BdG level [LR-MCTDHB $(M=1)$ ]. Table I shows that excitation 1 is already converged at the BdG level and that excitation 2 converges nicely with $M$. The third excitation, $2^{\prime}$, is first uncovered at the $\operatorname{LR}-\operatorname{MCTDHB}(M=2)$ level, i.e., it cannot be found within BdG theory. Table I shows that it converged nicely with $M$. The physical nature of this excitation is further discussed below. The next two excitations, 3 and $3^{\prime}$, change their relative position between Figs. 1 and 3, which makes it harder to see that they converge. This is particularly the case for excitation $3^{\prime}$, which is first uncovered at the LR$\operatorname{MCTDHB}(M=2)$ level. By examining Table I we see the convergence of these excitations as well.

From Table I we see that the higher excited 3 and more so $3^{\prime}$ states converge slower as a function of $M$ than the lower excitations. This is a general tendency expected for any manybody method. Yet, there is more to that than merely energetics. In the harmonic trap, the physical nature of the excitations is either c.m. or rel excitations. Excitation $3^{\prime}$ (as is excitation $2^{\prime}$ ) is a c.m. excitation, a higher harmonic of excitation 1.

Generally, the c.m. excitations converge slower than the rel excitations; see Table I. There are two reasons for that: (i) except for the fundamental excitation 1, these excitations are not described at the $M=1(\mathrm{BdG})$ level, so to start with one needs more orbitals to describe them, and (ii) they consist of excitations of a single collective particle. The MCTDHB ground-state wave function is constructed in the laboratory frame, and requires quite a few self-consistent orbitals to faithfully represent the c.m. coordinate for these higher excitations. Fortunately, the energies of the c.m. excitations are analytically known - they are (in units of the trap frequency $\left.\omega_{0}\right)$ just the integers $1,2,3, \ldots$, which allows us to assess

TABLE I. Frequencies of the excitations of $N=10$ bosons in the harmonic potential (in units of the trap frequency $\omega_{0}$ ) as a function of $M$. The results are taken from Figs. 1 and 3. Convergence of LR-MCTDHB is now clearly seen. Excitations $2^{\prime}$ and $3^{\prime}$ are first uncovered at the LR-MCTDHB $(M=2)$ level, i.e., they cannot be found (n/a) within BdG theory. The type of excitations-“c.m." and "rel"-is indicated. The exact frequencies of the c.m. excitations are analytically known.

\begin{tabular}{lccccrr}
\hline \hline Excitation No. & $M=1$ & $M=2$ & $M=4$ & $M=5$ & Type & Exact analytical \\
\hline 1 & 1.00000000 & 1.00000000 & 1.00000000 & 1.00000000 & c.m. & 1.00000000 \\
2 & 1.93782050 & 1.93549034 & 1.93344998 & 1.93335009 & rel & c.m. \\
$2^{\prime}$ & n/a & 2.04948358 & 2.00990796 & 2.00785285 & 2.00000000 \\
3 & 2.90297701 & 2.90431103 & 2.90163006 & 2.90254983 & rel & c.m. \\
$3^{\prime}$ & n/a & 2.81478928 & 2.94532236 & 2.98629877 & 3.00000000 \\
\hline \hline
\end{tabular}




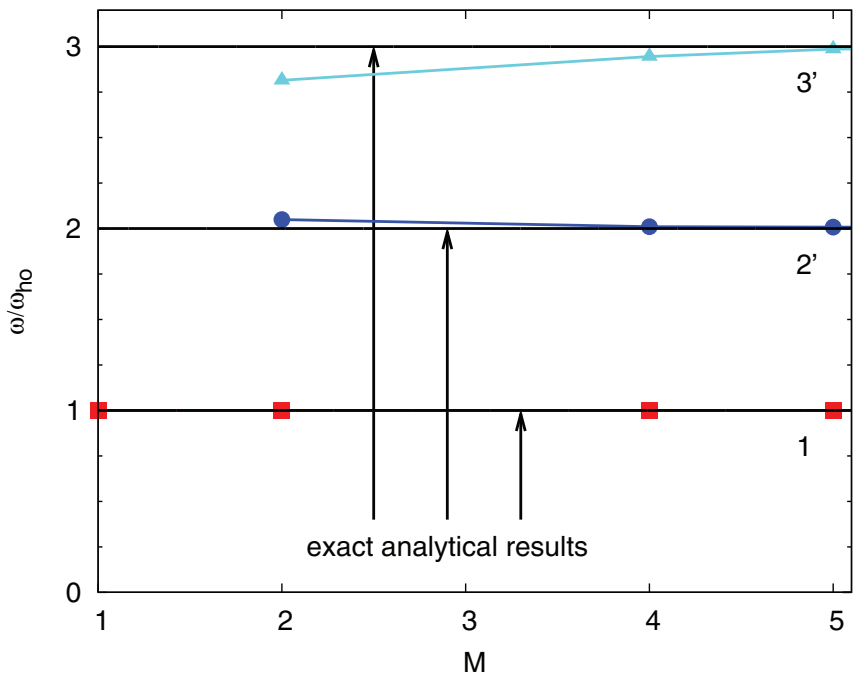

FIG. 4. (Color online) Convergence of the center-of-mass excitations of $N=10$ bosons in the harmonic potential as a function of $M$ (data combined from Figs. 1 and 3). The horizontal lines are the exact analytical values. All quantities are dimensionless.

their convergence (the rel excitations depend on the interaction strength and are generally not analytically known). Figure 4 depicts as a function of $M$ the frequencies of the c.m. excitations and follows their convergence-to the analytically known results.

Here it is important to mention that the $\operatorname{MCTDHB}(M)$ method is a variational theory $[21,42,43]$ - the variational principle guarantees that the approximate solution converges to the exact one with $M$ (also see [44]). Moreover, the total energy approaches the exact value from the above. The linear-response part of the $\operatorname{LR}-\operatorname{MCTDHB}(M)$ method is not variational, implying that the positions of the lines in the linear-response excitation spectra can be above as well as below the exact values; see for an example excitations $2^{\prime}$ and $3^{\prime}$ in Fig. 4, respectively. However, as a general prescription, to get numerically converged results for highly excited states within a linear-response method one has to provide a very good ground many-body wave function to start from.

Let us now address a general aspect of the LR-MCTDHB theory, namely, the above-used decomposition of the LRMCTDHB excitation eigenvectors into orbitals' and CI components, according to Eq. (75). Clearly, if the number $M$ of self-consistent orbitals used tends to infinity, then one gets the exact results already within the CI part, because the complete Fock space is spanned there. In this case there is no need to use self-consistent (time-adaptive) basis sets. Within the above-defined nomenclature for orbitals' and CI contributions, it would mean that the orbital part of the linear-response equations does not contribute at all. Conversely, a nonzero contribution from the orbital part means that self-consistency is still desirable. Indeed, a close inspection of the gray bars plotted in Figs. 1 and 3 supports this conjecture. In this respect it is especially interesting and instructive to analyze the 3 and $3^{\prime}$ excitations of the LR-MCTDHB(2) spectrum. They have structures, involving excitations to the one-particle orbitals with two and three nodes; see Fig. 2 and associated discussion in Sec. IV A1. However, within the Fock subspace spanned by the $\operatorname{MCTDHB}(M=2)$ theory the needed CI excitations are in principle unavailable, because all permanents are constructed by permuting bosons among two single-particle functions (orbitals). Neither of these orbitals has the required twoor three-node structure: the first orbital has no nodes, the second orbital has only a one-node profile. Nevertheless, the linear-response on top of the $\mathrm{MCTDHB}(2)$ theory provides us with these excitations. As expected, they have the orbitaldominating structure, i.e., the contribution from the CI part is very small.

Concluding, the LR-MCTDHB $(M)$ equations provide improved description of the excitations which are poorly spanned by or even completely out of the $\operatorname{MCTDHB}(M)$ Fock subspace. The LR-MCTDHB $(M)$ provides converged results on excited states with increasing $M$. To get better excited states one has to provide a better initial state.

\section{B. Applications to Bose-Einstein condensates in shallow double-well potentials}

In the preceding discussions we have seen that even in a simple harmonic well, where the BEC exhibits small fragmentation, our many-body response theory predicts new excitations, not described by the standard LR-GP approach. We now calculate the many-body excitation spectra of BoseEinstein condensates trapped in a shallow symmetric onedimensional double-well potential

$$
V(x)=b / 2 \cos \left(\frac{\pi}{3} x\right)+\omega_{h o}^{2} x^{2} / 2,
$$

with $b=5$ and $\omega_{h o}=\sqrt{2}$, for the same systems of $N=10$ and $N=100$ bosons with $\lambda_{0} N=1$. This is an intricate, delicate, and interesting problem, because in this regime of the parameters the double well is so shallow that the spatial modes are still spatially strongly overlapping, excluding thereby the applicability of, e.g., the Bose-Hubbard theory. Remarkably, the ground-state fragmentation in this case is still very small-it is about $0.2 \%$ and $0.03 \%$ for the systems of $N=10$ and $N=100$ bosons, respectively. Hence the popular LR-GP theory is the main source of information on excited states available in such systems. Let us see how the LR-MCTDHB results change the BdG excitation picture.

\section{Results for $N=100$ and $N=10$}

The LR-GP (BdG) and LR-MCTDHB(2) excitation spectra for the systems of $N=100$ (lower two panels) and $N=10$ (upper two panels) bosons trapped in the shallow double well are depicted in Fig. 5. The GP and $\operatorname{MCTDHB}(2)$ densities of the ground state are very similar and schematically shown in the insets together with the trapping potential. The ground-state density is, because of the central barrier, broader than in the harmonic case, but there is no spatial separation of the modes at this barrier height. The $x$ axes in Fig. 5 indicate excitation energies in units of the envelope trap's frequency $\omega_{h o}$; see Eq. (77). The depicted response weights $\gamma_{k}$ and state characterizations and attributions $t_{k}$ are computed and performed, respectively, in the same way as is done in the above-studied harmonic case. The symmetry 


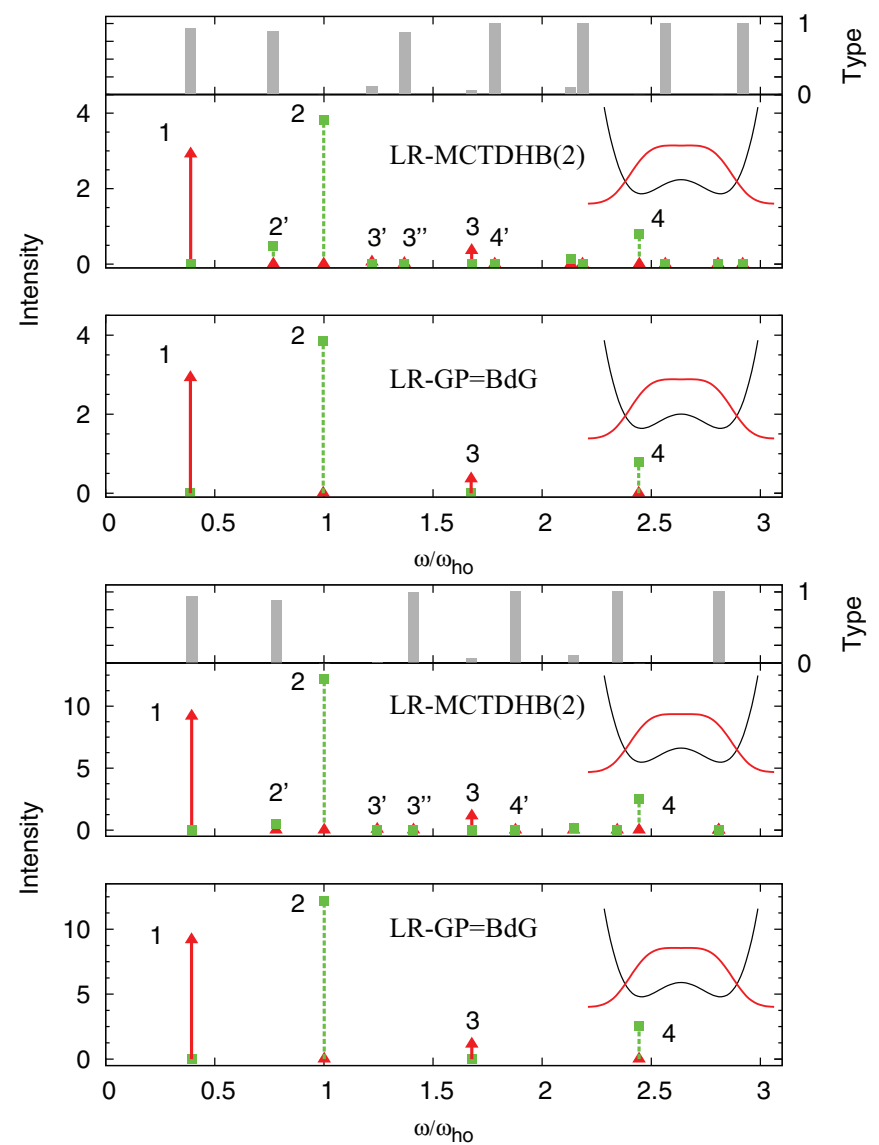

FIG. 5. (Color online) Comparison of the standard LR-GP (BdG) and LR-MCTDHB(2) linear-response spectra computed for the systems of $N=10$ (upper two panels) and $N=100$ (lower two panels) bosons trapped in a very shallow one-dimensional double-well potential, Eq. (77), and subject to linear and quadratic perturbations. The trap potential and ground-state density (red solid line) are shown in the insets. The depicted response weights and the scheme of the state enumeration and characterizations are the same as in the harmonic case; see Fig. 1 and text. The key observation here is that the number of low-lying excitations appearing on the many-body level and not described by the BdG theory is much larger in comparison with the harmonic-trap results. All quantities are dimensionless.

of the double-well trap potential allows us to study odd- and even-parity excitations separately, so we use solid (red) lines with triangles to mark the odd excitations and dashed (green) lines with squares to depict the even ones.

We first compare the LR-GP and LR-MCTDHB(2) excitation spectra for the system of $N=10$ bosons. The main observation is that the many-body spectrum has more spectral features in comparison with the LR-GP mean-field-based spectrum. The second observation is that the spectral lines, marked as 1,2,3,4, are similarly described by both the standard LR-GP and LR-MCTDHB methods. In Fig. 6 we plot the LR-GP and LR-MCTDHB density responses corresponding to several low-lying spectral lines including these ones. Similar to the harmonic case, the most intense spectral lines can be attributed to single-particle $(1 h-1 p)$ excitations from the condensate to higher excited modes. The LR-GP and LR-MCTDHB results for these excitations have very similar energetics and response weights, as well as density responses.

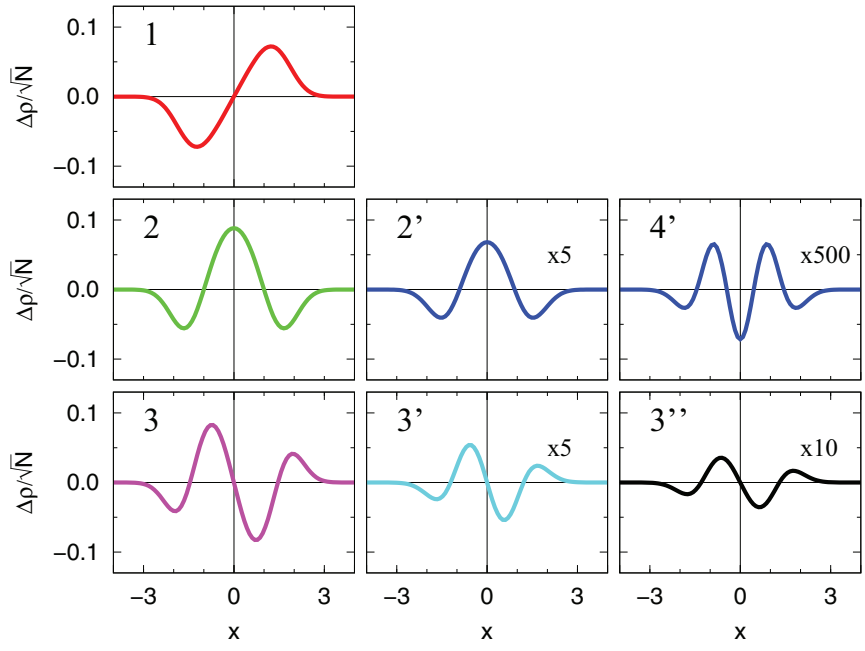

FIG. 6. (Color online) Density responses for $N=10$ atoms in a very shallow one-dimensional double-well potential, Eq. (77). Plotted are the real parts of the response densities $\Delta \rho(x)=\operatorname{Re}\left[\Delta \rho_{o}^{k}(x)+\right.$ $\Delta \rho_{c}^{k}(x)$ ] corresponding to real-valued $\gamma_{k}$ 's; see discussion in the text. All the excitations are numerated according to the underlying nodal structures (number of nodes) in the respective response density. The LR-GP (BdG) results for the first few excitations are depicted in the left column of the figure. The LR-MCTDHB results for these excitations are quite similar and, therefore, not shown. The manybody excitations, not describable by the BdG theory, are labeled by the numbers with a prime, and magnified for better visibility. All quantities are dimensionless.

However, there are several very important and significant differences between the mean-field-based and many-body linear-response predictions. First of all, according to the LR-GP theory the lowest-in-energy excitation of the gerade symmetry is at about 1 unit of energy and corresponds to a single-boson transfer from the condensate to the secondexcited two-node mode. In contrast, our many-body theory predicts that the lowest-in-energy response to the gerade perturbation takes place at 0.75 units of energy and corresponds to a many-body excited state, where two bosons are transferred from the condensate to the one-node mode. The next observable difference is that in between the mean-fieldbased excited states labeled as 2 and 3 there are two many-body excited states labeled as $3^{\prime}$ and $3^{\prime \prime}$. There are even more excitations lying in the energy window between the third and fourth mean-field-based excited states.

The tendency concerning the density responses of twoparticle excitations observed in the harmonic case also persists in shallow double wells. In Fig. 6 one can see that the non-mean-field-based excitations, marked as $2^{\prime}$ and $3^{\prime}$ and corresponding to two-boson $(2 h-2 p)$ excitations, provide smaller density responses to external perturbations in comparison with their $1 h-1 p$ counterparts. The higher excitations can also be characterized according to their nodal structure; see the excitations labeled $3^{\prime \prime}$ and $4^{\prime}$ in Fig. 6. However, their density responses are even weaker-we have to magnify them significantly for better visibility. We conjecture that other than one-body response operators will have to be considered in order to activate such excitations more efficiently. 
Let us now compare the $N=10$ and $N=100$ spectra depicted in Fig. 5. Since we used the same nonlinearity $\lambda_{0} N=$ 1 for both systems, the position of the LR-GP lines and their relative intensities are the same. The LR-MCTDHB(2) spectra of these systems, however, reveal some differences. First of all, there are small differences in the relative intensity and position of the lowest-in-energy many-body spectral line labeled as $2^{\prime}$. There are also small differences in the positions of the spectral lines corresponding to higher non-mean-field-based excited states. The differences between the excitation spectra of the $N=10$ and $N=100$ systems become more pronounced at higher energies.

Summarizing, the LR-MCTDHB theory predicts that the excitation spectrum of a condensate in a shallow double well possesses some additional spectral features not described by the standard LR-GP theory. In particular, the energy of the lowest-in-energy excitation of even symmetry is almost $25 \%$ lower than predicted by LR-GP theory. Moreover, this excitation is not of a single-particle nature as predicted by LR-GP theory, but it rather consists of a transfer of two bosons from the condensate to the lowest ungerade mode. Generally, in shallow double-well systems the number of low-lying non-mean-field-based states is larger in comparison with the harmonic case. The existence of low-lying excited states not described by the LR-GP theory can have very important consequences on the quantum dynamics and temperature properties of ultracold systems trapped in unharmonic potentials.

\section{Including more modes $(N=10$ and $M=4,5)$}

Now our goal is to investigate the convergence of the LR-MCTDHB $(M)$ results for double-well traps. Again, to make the computations more feasible we consider the system of $N=10$ bosons and compare the LR-MCTDHB $(M)$ spectra for $M=1,2,4,5$. The LR-MCTDHB(1) $\equiv$ LR-GP and LR-MCTDHB(2) results are depicted in Fig. 5; the LRMCTDHB(4) and LR-MCTDHB(5) spectra are plotted in Fig. 7.

In Fig. 7 the low-lying parts of the LR-MCTDHB(4) and LR-MCTDHB(5) spectra are identical, indicating the convergence of the results. As expected, differences start to appear at higher excitation energies and become more pronounced in the energy window between 2 and 3 units of energy. It is clear that new excitations which appear due to the inclusion of more orbitals would need even more orbitals to converge.

Summarizing, the LR-MCTDHB method is capable of providing converged results for excited states of a BEC in shallow double wells. The LR-MCTDHB method provides better description of the low-lying excited states than the highly excited ones at a given level of theory $M$; in order to obtain numerically converged results for higher excited states one has to perform the computation with a better initial state, i.e., to use higher levels of the $\operatorname{MCTDHB}(M)$ theory.

\section{SUMMARY AND CONCLUDING REMARKS}

To explore the excitation spectrum of trapped interacting bosons we derived a general many-body linear-response theory obtained from linearization of the MCTDHB equations. We have applied the developed LR-MCTDHB theory to study
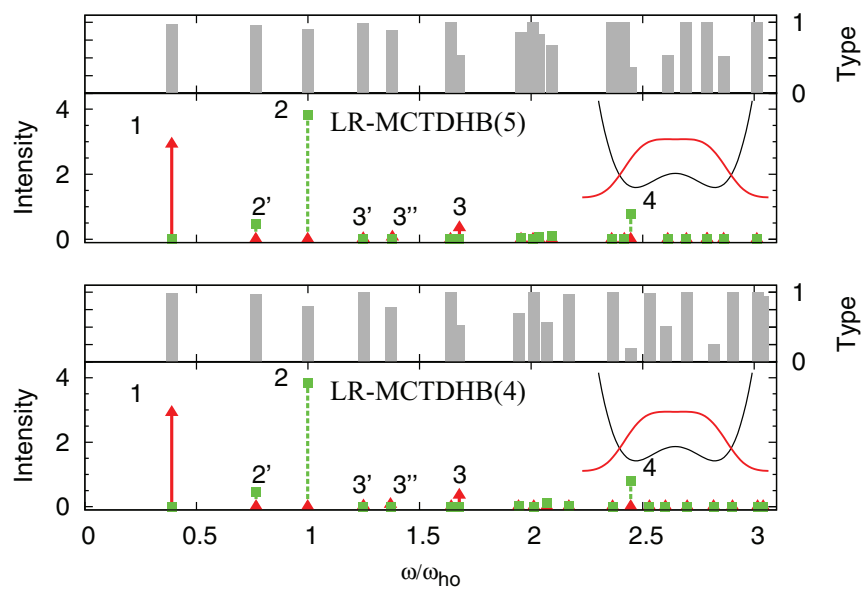

FIG. 7. (Color online) Convergence of LR-MCTDHB $(M)$ theory. Comparison of the linear-response spectra for $N=10$ bosons in a very shallow one-dimensional double-well potential, Eq. (77), and subject to linear and quadratic perturbations. The depicted LR-MCTDHB(4) and LR-MCTDHB(5) response weights and state characterizations are obtained in the same way as in Fig. 1; see text for more details. The main observation is that the lowest-in-energy excitations are numerically converged already at a low level of the LR-MCTDHB $(M)$ theory. By increasing $M$ we improve the quality of description of the higher-energy excitations as well. All quantities are dimensionless.

excitations of BECs trapped in the harmonic and shallow double-well traps.

The LR-MCTDHB theory consists of a linear-response matrix which accounts for the coupled response of the orbitals and state vector. This is reflected in the existence of response amplitudes for both the orbitals and CI coefficients, which are associated with the same excitation frequency. Similar to the linear-response theories of fully condensed (LR-GP [62]) and fully fragmented (LR-BMF [31]) BECs, the orbitals' response amplitudes are orthogonal to all the ground-state orbitals. The response matrix can be divided into submatrices. One accounts for the orbitals, and can be considered as the generalization of the linear-response matrix of LR-BMF. Another one accounts for the CI coefficients and contains the CI Hamiltonian in it. The coupling between the orbitals and the state vector in the response matrix is through the off diagonals. The response weights and the density response have also been derived, and we find that they are sums of the orbitals' and coefficients' parts.

To obtain excitation spectra we first calculate the ground state. This is done at a certain level $M$ of the $\operatorname{MCTDHB}(M)$ equations. Thereafter, we explicitly construct the linearresponse matrix, Eq. (60), and diagonalize it numerically, solving thereby the eigenvalue problem Eq. (63). The obtained eigenvalues give the excitation energies while the eigenvectors are used to compute the response weights and density responses. To shed more light on the nature of the excitations we have analyzed the underlying structure of the respective eigenvectors in terms of the relative contributions of the orbitals' and coefficients' parts. In the harmonic potential we analyzed the excitations in terms of center-of-mass and relative-motion degrees of freedom. 
The response matrix which provides the desired excitation energies does not depend on the special form of the perturbing field. The choice of the perturbing field can be utilized to study the nature of the excited states. Here, we have chosen $x$ and $x^{2}$ to distinguish between odd or even and dipole or quadruple transitions. One can easily envision additional perturbing fields to characterize further the spectrum of the excited states under inspection.

We have contrasted the predictions of the standard LRGP (BdG) and our many-body LR-MCTDHB theories in the situation where the initial ground state is essentially completely condensed, i.e., the GP and LR-GP theories are believed to provide adequate descriptions. The LR-MCTDHB reproduces and improves the mean-field-based excitations and, also, predicts additional many-body excited states which are out of the realm of the mean-field-based linear response. In particular, we were able to calculate the excitation energy of the higher harmonic of the dipole excitation in a harmonic trap. In the shallow double-well system the excitation of the same nature turns out to be the lowest-in-energy gerade excitation. Generally, in shallow double-well systems the number of low-lying non-mean-field-based excitations is larger in comparison to the harmonic case. Consequently, the existence of low-lying excited states that are not described by the LR-GP (BdG) theory can have very important consequences for the quantum dynamics and temperature properties of trapped ultracold bosonic systems, especially in unharmonic potentials.
We have also assessed the convergence of the LR$\operatorname{MCTDHB}(M)$ equations by direct comparison of the response spectra computed at different levels $M=1,2,4,5$ of the theory. The convergence is well achieved for low-lying excitations. However, in order to obtain numerically converged results for higher excited states, one faces a growing numerical effort because higher levels of the $\operatorname{MCTDHB}(M)$ theory have to be used to provide the very good ground many-body wave function needed from which to start. Hence future implementations will be based on efficient strategies for solving the MCTDHB equations [67].

In conclusion, we derived a theory for excitation spectra of trapped interacting Bose systems beyond the available approaches. Our first results based on the application of this new method suggest that it has a vast perspective to predict excitations also in BECs with attractive interactions or dipolar interactions.

\section{ACKNOWLEDGMENTS}

J.G. appreciates support from the Alexander von Humboldt Foundation. We acknowledge financial support by the DFG also within the framework of the Enable fund of the excellence initiative at Heidelberg University, the HGS MathComp, a Minerva Short Term research grant, and the STREP project "QIBEC". Computation time on the bwGRiD and the Cray XE6 cluster Hermit at the HLRS in Stuttgart are gratefully acknowledged.
[1] R. Folman, P. Krüger, J. Schmiedmayer, J. Denschlag, and C. Henkel, Adv. At. Mol. Opt. Phys. 48, 263 (2002).

[2] R. Grimm, M. Weidemüller, and Y. Ovchinnikov, Adv. At. Mol. Opt. Phys. 42, 95 (2000).

[3] T. Schumm, S. Hofferberth, L. M. Andersson, S. Wildermuth, S. Groth, I. Bar-Joseph, J. Schmiedmayer, and P. Krüger, Nature Phys. 1, 57 (2005).

[4] M. Albiez, R. Gati, J. Folling, S. Hunsmann, M. Cristiani, and M. K. Oberthaler, Phys. Rev. Lett. 95, 010402 (2005).

[5] S. Levy, E. Lahoud, I. Shomroni, and J. Steinhauer, Nature (London) 449, 579 (2007).

[6] J. Estève, C. Gross, A. Weller, S. Giovanazzi, and M. K. Oberthaler, Nature (London) 455, 1216 (2008).

[7] K. Maussang, G. E. Marti, T. Schneider, P. Treutlein, Y. Li, A. Sinatra, R. Long, J. Estève, and J. Reichel, Phys. Rev. Lett. 105, 080403 (2010).

[8] R. Bücker, J. Grond, S. Manz, T. Berrada, T. Betz, C. Koller, U. Hohenester, T. Schumm, A. Perrin, and J. Schmiedmayer, Nature Phys. 7, 608 (2011).

[9] S. Will, T. Best, U. Schneider, L. Hackermüller, D.-S. Lühmann, and I. Bloch, Nature (London) 465, 197 (2010).

[10] M. J. Mark, E. Haller, K. Lauber, J. G. Danzl, A. J. Daley, and H.-C. Nägerl, Phys. Rev. Lett. 107, 175301 (2011).

[11] N. N. Bogoliubov, J. Phys. USSR 11, 23 (1947) (reprinted in D. Pines, The Many-body Problem, W. A. Benjamin, New York, 1961).

[12] F. Dalfovo, S. Giorgini, L. P. Pitaevskii, and S. Stringari, Rev. Mod. Phys. 71, 463 (1999).
[13] A. Leggett, Rev. Mod. Phys. 73, 307 (2001).

[14] C. J. Pethick and H. Smith, Bose-Einstein Condensation in Dilute Gases, 2nd ed. (Cambridge University Press, Cambridge, England, 2008).

[15] O. Penrose and L. Onsager, Phys. Rev. 104, 576 (1956).

[16] P. Nozières and D. Saint James, J. Phys. France 43, 1133 (1982).

[17] P. Nozières, in Bose-Einstein Condensation, edited by A. Griffin, D. W. Snoke, and S. Stringari (Cambridge University Press, Cambridge, UK, 1996).

[18] K. Xu, Y. Liu, D. E. Miller, J. K. Chin, W. Setiawan, and W. Ketterle, Phys. Rev. Lett. 96, 180405 (2006).

[19] E. J. Mueller, T.-L. Ho, M. Ueda, and G. Baym, Phys. Rev. A 74, 033612 (2006).

[20] R. W. Spekkens and J. E. Sipe, Phys. Rev. A 59, 3868 (1999).

[21] A. I. Streltsov, O. E. Alon, and L. S. Cederbaum, Phys. Rev. A 73, 063626 (2006).

[22] K. Sakmann, A. I. Streltsov, O. E. Alon, and L. S. Cederbaum, Phys. Rev. A 78, 023615 (2008).

[23] O. E. Alon and L. S. Cederbaum, Phys. Rev. Lett. 95, 140402 (2005).

[24] A. I. Streltsov, L. S. Cederbaum, and N. Moiseyev, Phys. Rev. A 70, 053607 (2004).

[25] M. Greiner, O. Mandel, T. Esslinger, T. W. Hänsch, and I. Bloch, Nature (London) 415, 39 (2002).

[26] Y. Shin, M. Saba, A. Schirotzek, T. A. Pasquini, A. E. Leanhardt, D. E. Pritchard, and W. Ketterle, Phys. Rev. Lett. 92, 150401 (2004).

[27] E. B. Kolomeisky, T. J. Newman, J. P. Straley, and X. Qi, Phys. Rev. Lett. 85, 1146 (2000). 
[28] D. S. Petrov, G. V. Shlyapnikov, and J. T. M. Walraven, Phys. Rev. Lett. 85, 3745 (2000).

[29] A. K. Tuchman, W. Li, H. Chien, S. Dettmer, and M. A. Kasevich, New J. Phys. 8, 311 (2006).

[30] G.-S. Paraoanu, S. Kohler, F. Sols, and A. J. Leggett, J. Phys. B 34, 4689 (2001).

[31] J. Grond, A. I. Streltsov, L. S. Cederbaum, and O. E. Alon, Phys. Rev. A 86, 063607 (2012).

[32] L. S. Cederbaum and A. I. Streltsov, Phys. Lett. A 318, 564 (2003).

[33] O. E. Alon, A. I. Streltsov, and L. S. Cederbaum, Phys. Lett. A 362, 453 (2007).

[34] D. Masiello, S. B. McKagan, and W. P. Reinhardt, Phys. Rev. A 72, 063624 (2005).

[35] S. Zöllner, H.-D. Meyer, and P. Schmelcher, Phys. Rev. A 75, 043608 (2007).

[36] D. van Oosten, P. van der Straten, and H. T. C. Stoof, Phys. Rev. A 63, 053601 (2001).

[37] Y. Japha and Y. B. Band, Phys. Rev. A 84, 033630 (2011).

[38] V. Gritsev, A. Polkovnikov, and E. Demler, Phys. Rev. B 75, 174511 (2007).

[39] C. Menotti and N. Trivedi, Phys. Rev. B 77, 235120 (2008).

[40] K. V. Krutitsky, J. Larson, and M. Lewenstein, Phys. Rev. A 82, 033618 (2010).

[41] M. Snoek, Phys. Rev. A 85, 013635 (2012).

[42] A. I. Streltsov, O. E. Alon, and L. S. Cederbaum, Phys. Rev. Lett. 99, 030402 (2007).

[43] O. E. Alon, A. I. Streltsov, and L. S. Cederbaum, Phys. Rev. A 77, 033613 (2008).

[44] A. U. J. Lode, K. Sakmann, O. E. Alon, L. S. Cederbaum, and A. I. Streltsov, Phys. Rev. A 86, 063606 (2012).

[45] K. Sakmann, A. I. Streltsov, O. E. Alon, and L. S. Cederbaum, Phys. Rev. Lett. 103, 220601 (2009).

[46] A. I. Streltsov, O. E. Alon, and L. S. Cederbaum, Phys. Rev. Lett. 100, 130401 (2008).

[47] A. I. Streltsov, O. E. Alon, and L. S. Cederbaum, Phys. Rev. Lett. 106, 240401 (2011).

[48] A. U. J. Lode, A. I. Streltsov, K. Sakmann, O. E. Alon, and L. S. Cederbaum, Proc. Natl. Acad. Sci. USA 109, 13521 (2012).
[49] J. Grond, J. Schmiedmayer, and U. Hohenester, Phys. Rev. A 79, 021603(R) (2009).

[50] I. Březinová, A. U. J. Lode, A. I. Streltsov, O. E. Alon, L. S. Cederbaum, and J. Burgdörfer, Phys. Rev. A 86, 013630 (2012).

[51] M. H. Anderson, J. R. Ensher, M. R. Matthews, C. E. Wieman, and E. A. Cornell, Science 269, 198 (1995).

[52] C. C. Bradley, C. A. Sackett, J. J. Tollett, and R. G. Hulet, Phys. Rev. Lett. 75, 1687 (1995).

[53] K. B. Davis, M.-O. Mewes, M. R. Andrews, N. J. van Druten, D. S. Durfee, D. M. Kurn, and W. Ketterle, Phys. Rev. Lett. 75, 3969 (1995).

[54] D. S. Jin, J. R. Ensher, M. R. Matthews, C. E. Wieman, and E. A. Cornell, Phys. Rev. Lett. 77, 420 (1996).

[55] M.-O. Mewes, M. R. Andrews, N. J. van Druten, D. M. Kurn, D. S. Durfee, C. G. Townsend, and W. Ketterle, Phys. Rev. Lett. 77, 988 (1996).

[56] M. R. Andrews, D. M. Kurn, H.-J. Miesner, D. S. Durfee, C. G. Townsend, S. Inouye, and W. Ketterle, Phys. Rev. Lett. 79, 553 (1997).

[57] A. L. Fetter and J. D. A. Walecka, Quantum Theory of Many-Particle Systems (McGraw-Hill, New York, 1971).

[58] L. P. Pitaevskii and S. Stringari, Bose-Einstein Condensation (Oxford University Press, Oxford, 2003).

[59] M. Edwards, P. A. Ruprecht, K. Burnett, R. J. Dodd, and C. W. Clark, Phys. Rev. Lett. 77, 1671 (1996).

[60] P. A. Ruprecht, M. Edwards, K. Burnett, and C. W. Clark, Phys. Rev. A 54, 4178 (1996).

[61] C. W. Gardiner, Phys. Rev. A 56, 1414 (1997).

[62] Y. Castin and R. Dum, Phys. Rev. A 57, 3008 (1998).

[63] B. D. Esry, Phys. Rev. A 55, 1147 (1997).

[64] A. I. Streltsov, K. Sakmann, O. E. Alon, and L. S. Cederbaum, Phys. Rev. A 83, 043604 (2011).

[65] P.-O. Löwdin, Phys. Rev. 97, 1474 (1955).

[66] A. J. Coleman and V. I. Yukalov, Reduced Density Matrices: Coulson's Challenge (Springer, Berlin, 2000).

[67] A. I. Streltsov, O. E. Alon, and L. S. Cederbaum, Phys. Rev. A 81, 022124 (2010). 